\title{
Science Progress and Research
}

\section{Review Article}

ISSN: 2635-0955

DOI: https://doi.org/10.52152/spr/2021.121

\section{Implementation of High Dose Rate Brachytherapy in Cancer Treatment}

\author{
Amir Shahabaz, Muhammad Afzal
}

Dept. of Physics, Islamia University of Bahawal Pur, Pakistan

*Corresponding Author: Amir Shahabaz, MSc, Dept of Physics, Islamia University of Bahawal Pur, Pakistan. Email : aamirshahbaz789@gmail.com

\begin{abstract}
A technique of radiation therapy delivery in which the radioactive sources are placed very close or even inside the target volume, is called Brachytherapy (BT). Brachytherapy is a type of radiation therapy. It destroys cancer cells by making it hard for them to multiply. In this technique, a radiation source is placed directly into or near to a tumor. High dose-rate brachytherapy is also known as HDR brachytherapy, or temporary brachytherapy. It is a type of internal radiotherapy. HDR was developed to reduce the risk of cancer recurrence while shortening the amount of time it takes to get radiation treatment. HDR also limits the dose of radiation (associated side effects) to surrounding normal tissue. The important benefits of HDR brachytherapy include extremely precise radiation therapy delivered internally, used alone or after surgery to help prevent cancer recurrence, convenient treatments that are usually pain-free, and a reduction in the risk of common short- and long-term side effects. Currently, tumor dose as well as doses of the surrounding normal structures can be evaluated accurately, and high-dose-rate brachytherapy enables three-dimensional image guidance. The biological disadvantages of high-dose rate were overcome by fractional irradiation. In the definitive radiation therapy of cervical cancer, high-dose-rate brachytherapy is most necessary. Most patients feel little discomfort during brachytherapy. There is no residual radioactivity when the treatment is completed. A patient may be able to go home shortly after the procedure, resuming his normal activities with few restrictions. An advantage of brachytherapy is to deliver a high dose to the tumor during treatment and save the surrounding normal tissues. High-dose-rate (HDR) brachytherapy has great promise with respect to proper case selection and delivery technique, because it eliminates radiation exposure, can be performed on an outpatient basis and allows short treatment times. Additionally, by varying the dwell time at each dwell position, the use of a single-stepping source allows optimization of dose distribution. As the short treatment times do not allow any time for correction of errors, and mistakes can result in harm to patients, so the treatments must be executed carefully by using HDR brachytherapy. Refinements will occur primarily in the integration of imaging (computed tomography, magnetic resonance imaging, intraoperative ultrasonography) and optimization of dose distribution and it is expected that the use of HDR brachytherapy will greatly expand over the next decade.
\end{abstract}

Research at DOI https://doi.org/10.52152/spr/2021.121 and can also be viewed on the Journal's website at www.sprjonline.com". 
Various factors in the development of well-controlled randomized trials addressing issues of efficacy, quality of life, toxicity and costs-versus-benefits will ultimately define the role of HDR brachytherapy in the therapeutic armamentarium. Surrounding healthy tissues are not affected by the radiation due to the ability to target radiation therapy at high dose rates directly to the tumor. Treatment to be delivered as an outpatient in as few as one to five sessions is also allowed by this targeted high dose approach. HDR brachytherapy is the most precision radiation therapy, even better than carbon ion therapy. At the time of invasive placement of the radiation source into the tumor area, brachytherapy requires skills and techniques of radiation oncologists.

Keywords: Brachytherapy, Cancer Treatment, radiation therapy, Oncology

\section{INTRODUCTION}

Sparing the surrounding normal tissues, brachytherapy has the advantage of delivering a high dose of radiation to the tumor. Its procedures were previously performed by inserting the radioactive material directly into the tumor by giving high radiation exposure to the physicians while performing the procedure. To increase accuracy and reduce the radiation hazards, manually loaded techniques were introduced. Afterloaded techniques, hollow needles, catheters, or applicators are inserted into the tumor and then loaded with radioactive materials. Remote-controlled insertions of sources were used to eliminate radiation exposure to visitors and medical personnel. In this technique, the patient is housed in a shielded room while the radiation therapist controls the treatment from outside the room. Transfer tubes connect needles, hollow applicators, or catheters to the radioactive material, which is stored in a protected area inside the HDR afterloader and embedded into the tumor. By remote control, the radiation source is driven through the transfer tubes and into the tumor. Remote controlled brachytherapy can be done by LDR (low-dose-rate), MDR (medium-dose-rate), or HDR (High-dose-rate) techniques. 100-300 $\mathrm{Gy} / \mathrm{hunits}$ is the usual dose rate employed in current HDR brachytherapy. The use of remote-controlled brachytherapy eliminates the hazards of radiation exposure, whether the type is LDR, MDR, or HDR brachytherapy). Taking only a few minutes, the use of HDR has the added advantage of the treatments allowing them to be given on an outpatient basis with minimal risk of applicator movement and minimal patient discomfort.

Additionally, by varying the dwell time at each dwell position, use of a single stepping source used in most modern HDR afterloaders allows optimization of dose distribution. It should not be used to substitute for a poorly placed implant. However, it should be emphasized that optimization can improve the dose distribution. The current issue with HDR brachytherapy is the transmission of techniques and skills to younger radiation oncologists. Many radiation oncologists are now accustomed to seeing only the virtual screen. These trends must be revolutionized and the education of young radiation oncologists is of vital importance. Nag and Samsami [1] have provided examples of inappropriate optimization strategies, which can lead to suboptimal dosimetry plans and clinical problems.

\subsection{Background:}

Brachytherapy (BT) is the administration of radiation therapy in which radioactive sources are placed adjacent to or within tumors or body cavities [2].With rapid dose fall-off in the surrounding normal tissue, a high radiation dose can be delivered locally to the tumour using Brachytherapy. BT, in the past, was mostly performed with radium or radon sources. Currently, the use of artificially produced radionuclides, such as $198 \mathrm{Au}, 60 \mathrm{Co}$, $137 \mathrm{Cs}$, 125I, 192Ir, 103Pd and $103 \mathrm{Ru}$, is rapidly increasing. Electronic BT is a relatively new technological approach in which the radiation source is not an encapsulated radioactive isotope (radioisotope $\mathrm{BT}$ ) but it is a miniature electronic $X$ ray source that produces low energy radiation at a high-doserate.

According to the implant loading technique, BT can be performed as:

- Manual loading

- Manual after loading

- Remote controlled afterloading

According to the location of the implant, BT may be:

- Superficial, in which sources are placed in contact with the skin or a skin tumour

- Intracavitary, in which sources are placed into natural body cavities, e.g. the uterine cavity 
- Interstitial, in which sources are placed into tissues or tumours, e.g. the prostate.

According to the method how to remove radioactive sources, BT implants can be:

- Temporary ( in which sources are inserted and later removed)

- Permanent ( in which sources are inserted and left in place for the remainder of their active life)

Based on the dose rate of the sources used, BT can be:

- Low dose rate (LDR)

- Medium dose rate (MDR)

- $\quad$ High dose rate (HDR)

- $\quad$ Pulsed dose rate (PDR) ${ }^{[3]}$

The dose rate is defined in International Commission on Radiation Units and Measurements (ICRU) Report 38. ${ }^{[4]}$ The usual dose rate delivered in practice is approximately 100-300 Gy/h. An HDR means more than 12 Gy/h, although an HDR means more than 12 $\mathrm{Gy} / \mathrm{h}$.

\subsection{Brachytherapy:}

Brachytherapy is a form of radiation therapy which is used to treat various types of cancers. This treatment involves surgically placing radioactive capsules, seeds or other implants directly in or near the cancerous tumor.

The implants emit radiation for a short time, sparing healthy surrounding tissues. Radiation from brachytherapy targets cancerous tissue only. Brachytherapy is also called internal radiation therapy. ${ }^{[5]}$

\subsection{Procedure of Brachytherapy:}

A prescribed dose of radiation is emitted from the radioactive materials implanted inside or next to the tumor. This radiation destroys the genetic makeup of cancer cells. Damaged cancer cells can't multiply and grow further, eventually they die off. A doctor will go over pre-surgical requirements with the patient. A Patients may require an imaging scan, such as an MRI (magnetic resonance imaging) or a CT (computed tomography).Stop taking medications, such as blood thinners like warfarin or non-steroidal anti-inflammatory drugs (NSAIDs) that affect blood clotting. Before the procedure, use a bowel preparation (enema). Do not drink or eat anything for several hours before going to the hospital. The procedure varies depending on the brachytherapy type and cancer. The patients will have anesthesia to relieve pain and discomfort. To place the radioactive materials, a doctor uses a catheter (small flexible tube) or applicator device. Brachytherapy may occur inside the tumor or near the tumor. The applicator or catheter may stay in place until you finish treatments. Patients will need to stay in the hospital while undergoing HDR brachytherapy. The patient's body is radioactive during this time. A patient could potentially expose other people to radiation for several weeks or months if he has permanent brachytherapy (permanent brachytherapy also known as LDR). Patients should limit their contact with young children and pregnant women as directed by the doctor, although the exposure risk is small.

\subsection{Types of Brachytherapy:}

There are different types of brachytherapy implants, out of which some remain within the body permanently. Doctors remove temporary implants after treatment and select the best treatment for a patient's specific needs. Brachytherapy implants include:

- Low-dose rate (LDR): LDR implants emit low doses of radiation for one to seven days. After treatment or leaving them in place, a doctor may remove the implants permanently.

- High-dose rate (HDR): HDR implants discharge high doses of radiation for 10 to 20 minutes. The doctor then removes the implant. Treatment times vary from twice a day for up to five days or once a week for up to five weeks.

- Permanent: Radioactive implants emit radiation continuously until no radiation is left in them. The implants or seeds, which are about the size of a grain of rice, remain in the patient's body. This treatment is also called seed implantation. ${ }^{[5]}$

\subsection{Difference between internal radiation and external radiation:}

Internal radiation therapy is another name for brachytherapy. To destroy the tumor, cancer doctors place radioactive materials inside the body from within. The entire treatment takes place internally. 
With external radiation therapy, a machine delivers beams of radiation energy through the skin to the tumor. It has a slightly higher chance of radiation exposure to surrounding healthy tissue and organs. For some cancers, external radiation is the only possible treatment. ${ }^{[5]}$

Brachytherapy is a form of radiation therapy used to treat various cancers. Treatment involves surgically placing radioactive seeds, capsules or other implants directly in or near the cancerous tumor.

The implants give off radiation for a short time. Radiation from brachytherapy targets cancerous tissue, sparing healthy, surrounding tissue. Brachytherapy is also called internal radiation therapy. ${ }^{[5]}$

\subsection{Effects of Brachytherapy:}

Brachytherapy is a highly effective in treatment of certain types of cancer. It's most effective on cancers that haven't spread, or metastasized. Internal radiation therapy takes place inside the body. Brachytherapy targets just the tumor, sparing nearby healthy tissue and organs. Most side effects improve as the radiation leaves your body, external radiation therapy. [5]

\section{Importance of HDR Brachytherapy}

2.1 Advantages and disadvantages of highdose-rate (HDR) compared with low-doserate (LDR) brachytherapy:

\section{Advantages}

\section{- Radiation protection}

Radiation exposure hazard for caregivers and visitors is eliminated by HDR. Without any fear of radiation exposure, caregivers are able to provide optimal patient care. Source preparation and transportation can be eliminated with the help of HDR. There is minimal risk of losing a radioactive source because there is only one source.

- Allows shorter treatment times

Since prolonged bed rest is eliminated, there is less patient discomfort. Those patients who are at high risk of pulmonary embolism due to prolonged bedding and also those patients who may not bear long durations of isolation and rest, so it is possible to treat such patients. During therapy, there is a minimum risk of applicator motion. As outpatient therapy is possible, there are reduced hospitalization costs. HDR may allow greater displacement of nearest normal tissues (by packing or retraction), which could potentially reduce morbidity. The institutes that have a high volume of brachytherapy patients can treat a larger number of patients but there are insufficient in-patient facilities (e.g., in some developing countries). HDR allows intraoperative treatments which are completed whereas the patient is still in the operating room.

- HDR sources are of smaller diameter than the Cesium sources that are used for intracavitary LDR

This reduces the need for heavy sedation or general anesthesia as this reduces the need for dilatation of the cervix. High-risk patients can be more safely treated who are unable to bear general anesthesia. HDR allows for interstitial, intraluminal and percutaneous insertions.

- HDR makes treatment dose distribution optimization possible.

The source positions allow for greater control of the dose distribution and potentially less morbidity, and an almost infinite variation of the effective source strengths is allowed by variations of the dwell times of a single stepping source.

\section{Disadvantages}

\section{- Radiobiological}

Multiple treatments are required because for the repair of sublethal damage in normal tissue, the redistribution of cells within the cell cycle, or reoxygenation of the tumour cells are allowed by the short treatment times.

- Limited experience

In the United States, few centers have longterm (greater than 20 years) experience. Until recently, standardized treatment guidelines were not available; however, guidelines for HDR have recently been provided by the American Brachytherapy Society at various sites. [6], [7]

- The economic disadvantage

As the remote after loaders cost approximately $\$ 300,000$, the use of HDR brachytherapy, compared with manual after loading techniques, demands a large initial capital expenditure. As the procedures are more labor intensive, there are additional costs for a shielded room, and personnel costs are higher.

- Greater potential risks

There is greater potential harm if the machine malfunctions or if there is a calculation error as a high activity source is used. The short treatment times, compared 
with LDR, allow much less time to detect and correct errors.

\subsection{Benefits of brachytherapy treatment}

There are a number of benefits of brachytherapy treatment that you may wish to consider when deciding the best treatment option for you. While compared to other cancer treatments, these benefits may make it a potential treatment option, such as surgery or external beam radiotherapy.

In treating cancer Brachytherapy is very effective, as the radiation is delivered with a high level of accuracy. It doesn't involve extensive surgery, so we can say that it is a minimally invasive technique. In many cases, it can be done as an outpatient procedure, avoiding the need for an overnight stay in the hospital. ${ }^{[8]}$ Due to the targeted and precise nature of delivering the radiotherapy from inside the body, brachytherapy has a minimized risk of side effects. ${ }^{[9]}$ Very short treatment duration (typically from 1 to 5 days) is required for brachytherapy. People can usually return to everyday activities very quickly because brachytherapy has short recovery times (typically 2 to 5 days). Brachytherapy requires fewer visits to the hospital and overnight stays than other options. ${ }^{[8],[10]}$
Side effects from brachytherapy vary depending on treatment type and the cancer being treated. These problems typically improve in a few months after treatment stops. You may experience:

1. Urinary incontinence or difficulty urinating

2. Erectile dysfunction

3. Fatigue

4. Fecal (bowel) incontinence, constipation or diarrhea

5. Hair loss

6. Headaches

7. Mouth sores

8. Vomiting and nausea

9. Cough or shortness of breath (dyspnea) ${ }^{[5]}$

\subsection{Comparison of Brachytherapy to other treatments}

More than one type of treatment may be given for some cancers. In combination with external beam radiotherapy (EBRT), brachytherapy can be used. This can help improve the side effects from the radiation dose overall and the effectiveness of the radiotherapy. ${ }^{[8]}$

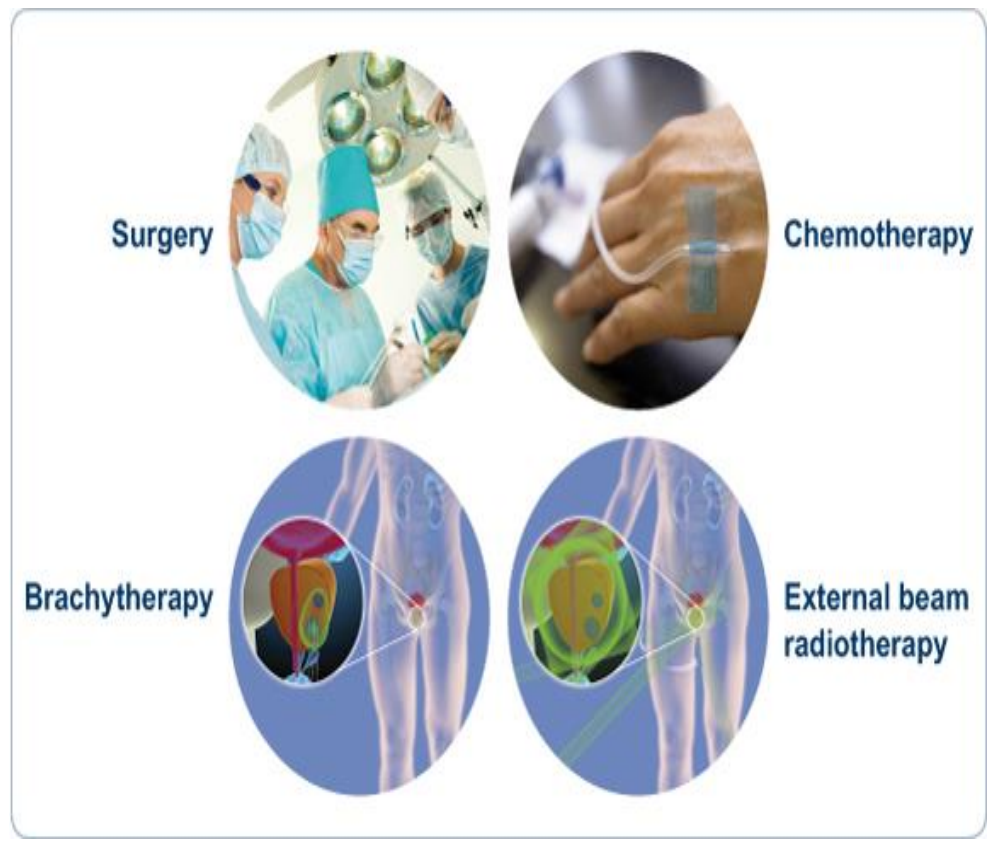

Figure 2.1: comparison of brachytherapy [10a]

In terms of the effectiveness of treatment, studies have shown that brachytherapy is comparable to external beam radiotherapy and surgery when treating many types of cancer. However, studies have shown that patients generally experience fewer side effects after brachytherapy than other treatment options. ${ }^{[9]}$

Now we are discussing comparison of Brachytherapy to other treatments as: 


\section{Brachytherapy}

- As the radiation is delivered with a high degree of precision from within the body, so brachytherapy is effective in treating many types of cancer.

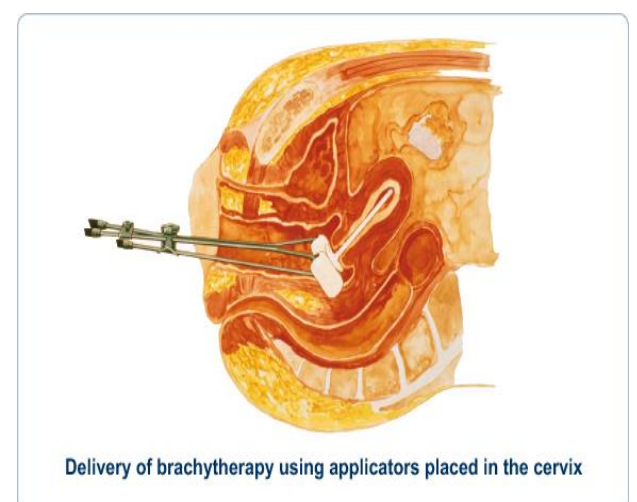

Figure 2.2: Delivery of Brachytherapy for cervix cancer ${ }^{[10 b]}$

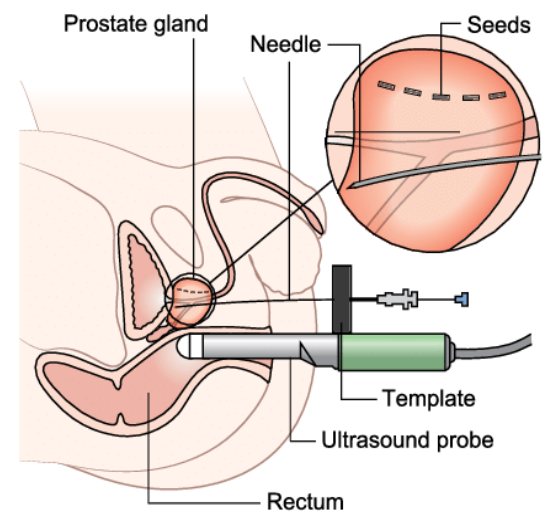

Figure 2.3: Brachytherapy for Prostate Cancer ${ }^{[10 \mathrm{c}]}$

- By reducing the risk of unnecessary damage to healthy tissues and organs close to the tumor radiation is precisely delivered from within the body. This helps reduce the risk of potential side effects. ${ }^{[8]}$

- Can be completed in 1-5 days in total as quick recovery times (typically 2 to 5 days); often on an outpatient basi. ${ }^{[10]}$

\section{External beam radiotherapy (EBRT);}

- As EBRT can be used almost anywhere in the body so it is effective in treating a wide variety of cancers.

- More healthy tissues and organs may be exposed to the radiation, as EBRT delivers radiation from outside the body and the radiation has to travel through healthy tissue to reach the tumor.
- As the radiation passes through healthy tissues, treatment has to be spaced out to limit damage to healthy cells, therefore typically completed over 6-8 weeks of small daily doses. ${ }^{[9,11]}$

Surgery

- Surgery is very effective in treating tumors that are accessible and have not spread to other parts of the body.

- Surgery can cause scarring and often requires longer recovery times whilst the wound heals, as it only affects the immediate area being operated on it.

- Surgery usually requires a stay in hospital and there is often a period of recovery time, as it is usually a one-time procedure. ${ }^{[12]}$

\section{Chemotherapy}

- As the treatment (drug) is administered to the whole body it is effective in treating cancer that has spread, so chemotherapy is effective in treating many types of cancer.

- A range of side effects are often experienced. As the chemotherapy drug is circulated throughout the whole body.

- Chemotherapy is usually given in cycles. This allows the cancer cells to be attacked at their most vulnerable time, and gives the body's normal cells time to recover. Each treatment cycle could last minutes, hours, or days, depending on the cancer being treated. ${ }^{[13]}$

\subsection{Treatment by Brachytherapy Brachytherapy can treat these cancers:}
1. Brain
2. Breast
3. Cervical
4. Eye
5. Gallbladder
6. Neck and neck
7. Lung
8. Prostate
9. Rectal
10. Skin
11. Thyroid
12. Uterine
13. Vaginal ${ }^{[5]}$

\section{Treatment of Cancer by Brachytherapy}

\subsection{A New HDR Protocol:}

Most radiation oncologists are familiar with LDR brachytherapy. LDR (at 30-50 cGy/h) can be added to external beam radiation therapy (EBRT) doses (at 2 Gy/day) to obtain equivalent total doses. HDR brachytherapy is a relatively 
new modality that is distinct from LDR brachytherapy, and radiation oncologists who are accustomed to LDR techniques must realize that experience in LDR cannot be automatically translated into expertise in HDR. It is important to review the current literature and survey the experiences of centers that have been performing HDR. When converting from LDR to HDR, one must keep the other parameters (chemotherapy, EBRT field/dose, dose-specification point, applicators, patient population, etc.) the same, changing only the LDR to HDR. Many radiation oncologists are not very familiar with the resultant biological effects, although fractionation schemes for HDR are widely variable. Nominal standard dose, time-dose factor or a dose reduction factor of 0.6 are Empirical methods that have been used in the past to convert HDR doses to LDR equivalent doses. The linear-quadratic (LQ) equation ${ }^{[14]}$ can be used to guide development of HDR doses and fractionation schedules. However, the LQ mathematical calculations are tedious and may not be practical on a day-today basis. ${ }^{[15]}$ Develop a simplified computer program to obtain the biologically equivalent doses for HDR. The clinician needs only to enter the EBRT total dose and dose/fraction and the number of HDR fractions. The equivalent doses for tumor and normal tissue effects will automatically be calculated by the computer program. Equivalent doses are expressed in clinically familiar terms (as if given at 2 Gy per fraction) rather than as biologically equivalent doses, which are unfamiliar to clinicians. Furthermore, a dose modifying factor (DMF) is applied to the normal tissues to account for the fact that doses to normal tissues are different from the doses to the tumor; this thing provides a more realistic equivalent normal tissue effect. This program can be used to determine HDR doses that are equivalent to LDR brachytherapy doses used to treat various cancers. Alternatively, the program may be used to express the equivalent dose of different HDR dose-fractionation regimes, as shown for cervical cancer in (Table 3.1). ${ }^{[16]}$ It is important to mention that the equivalent doses for tumor effects for the various fractionation regimes used for early-stage cervical cancers are so similar, ranging from $82 \mathrm{~Gy}$ to $85 \mathrm{~Gy}$, while those ones applied for advanced cancers are about 90 Gy (Table 3.1). The equivalent dose for normal tissue late effects depends on the assumed DMF $(0.6,0.7$, or 0.9$)$. If the doses to normal tissues were 60,70 , or $90 \%$ of the prescribed dose to point $\mathrm{A}$, the equivalent late effect on normal tissue (bladder or rectum) would be 59.5, 71 , or $98 \mathrm{~Gy}$, respectively for the fractionation scheme shown in (Table 3.1), row 1. ${ }^{[16]}$ Although the LQ biomathematical model can be helpful in determining equivalent doses, it has many limitations that must be kept in mind when using the program. The L-Q model accounts for the repair of sublethal damage and does not account for reoxygenation of hypoxic cells, reassortment within the cell cycle, or repopulation of tumor cells.

These factors are generally small under normal circumstances. However, large doses per fraction do not allow reoxygenation of hypoxic tumor cells or reassortment of tumors from radioresistant $\mathrm{S}$-phase. A large radiation dose preferentially kills radiosensitive cells, leaving a high number of hypoxic, radioresistant cells. Therefore, the computer program overestimates the tumor effect of a single large dose per fraction unless a resensitization factor is introduced.

Proliferation of tumor cells cannot took into account by the LQ equation. As the treatments are performed over a short duration, this factor is small. The LQ model will overestimate the actual tumor effect if the treatments are highly protracted (e.g., there is a long time interval between EBRT and HDR), or in cases of tumors with high proliferation rates, however the individual $\alpha / \beta$ values are very variable. The $\alpha / \beta$ values for early reactions vary from 6 to 13 (the default in the program is set at 10 ); the $\alpha / \beta$ values for late reactions vary from 1 to 7 (default being set at 3 ), while $\alpha / \beta$ values for tumors vary from 0.4 to 13 (the default being set at 10 ). The $\alpha / \beta$ values may vary even within the same tissue however, for a particular patient the values are not known. The equivalent doses obtained will, therefore, depend on the $\alpha / \beta$ values used for that particular calculation. The LQ model assumes complete repair between fractions. If the time interval between fractions is too short $(<6 \mathrm{~h})$ or the half-time of repair is very long, the repair of normal tissues will be incomplete, and the LQ formula will underestimate the biological effect. Hence, it is important to have a sufficient time interval (at least $6 \mathrm{~h}$ ) between treatment fractions. The infinite variation of the dwell times that is possible with HDR (or pulsed dose rate) allows better optimization of the doses than can be achieved with LDR. Better packing or retraction of normal tissues is possible with HDR, due to the short treatment duration. This factor is not usually taken into account in the LQ model (unless the DMF is altered). Another difference not accounted for in the LQ model is that the dose stated in brachytherapy is generally the minimum tumor dose. The doses within the tumor are much higher. Hence, the effective dose (for tumor control probability) is much higher for brachytherapy than for EBRT. ${ }^{[16]}$

In view of the many limitations of the LQ model, it must be stressed that, as with any 
mathematical model, the LQ model should be used judiciously as a guide only and should always be correlated with clinical judgment and outcome results. Caution is especially warranted whenever large fraction sizes are used, since their clinical results have not been well studied.

Table 3.1. American Brachytherapy Society suggested doses of external beam radiation therapy (EBRT) and high-dose-rate (HDR) brachytherapy to be used in treating early and advanced cervical cancer. The $\alpha / \beta$ ratio assumed for tumor equals ten. The $\alpha / \beta$ ratio assumed for normal tissue late effects equals three. $\mathrm{DMF}=$ dose modifying factor ${ }^{[16]}$

\begin{tabular}{|c|c|c|c|c|c|c|}
\hline $\begin{array}{l}\text { Total } \\
\text { EBRT } \\
\text { dosy (Gy) } \\
@ 1.8 \\
\text { Gy/fracti } \\
\text { on }\end{array}$ & $\begin{array}{l}\text { No. of } \\
\text { HDR } \\
\text { fractions }\end{array}$ & $\begin{array}{l}\text { HDR } \\
\text { dose/frac } \\
\text { tion (Gy) }\end{array}$ & $\begin{array}{l}\text { Equivale } \\
\text { nt dose } \\
\text { (Gy) for } \\
\text { tumor } \\
\text { effects }\end{array}$ & $\begin{array}{l}\text { Equivale } \\
\text { nt dose } \\
\text { (Gy) for } \\
\text { late } \\
\text { effects } \\
\text { with } \\
\text { DMF=0.6 }\end{array}$ & $\begin{array}{l}\text { Equivale } \\
\text { nt dose } \\
\text { (Gy) for } \\
\text { late } \\
\text { effects } \\
\text { with } \\
\text { DMF=0.7 }\end{array}$ & $\begin{array}{l}\text { Equivale } \\
\text { nt dose } \\
\text { (Gy) for } \\
\text { late } \\
\text { effects } \\
\text { with } \\
\text { DMF=0.9 }\end{array}$ \\
\hline \multicolumn{7}{|c|}{ Early cervical cancer } \\
\hline 19.8 & 6 & 7.5 & 85.1 & 59.5 & 71 & 98 \\
\hline 19.8 & 7 & 6.5 & 82 & 56.7 & 67.1 & 91.5 \\
\hline 19.8 & 8 & 6 & 83.5 & 57 & 67.4 & 91.6 \\
\hline 45 & 5 & 6 & 84.3 & 67 & 73.4 & 88.6 \\
\hline 45 & 6 & 5.3 & 84.8 & 66.8 & 73.1 & 87.7 \\
\hline \multicolumn{7}{|c|}{ Advanced cervical cancer } \\
\hline 45 & 5 & 6.5 & 88.9 & 70.1 & 77.6 & 95 \\
\hline 45 & 6 & 5.8 & 90.1 & 70.3 & 77.6 & 94.7 \\
\hline 50.4 & 4 & 7 & 89.2 & 72.6 & 79.4 & 95.3 \\
\hline 50.4 & 5 & 6 & 89.6 & 72.1 & 78.6 & 93.7 \\
\hline 50.4 & 6 & 5.3 & 90.1 & 72 & 78.3 & 92.9 \\
\hline
\end{tabular}

\subsection{Common Uses of HDR Brachytherapy:}

Although HDR brachytherapy has been used in almost every site in the body, it is most commonly used to treat cancers of the cervix, endometrium, lung, and esophagus. The prostate, bile duct, breast, brain, rectum, head and neck, skin, soft tissues, and blood vessels (coronary and peripheral arteries) areless common treated sites for HDR. It is generally used as a component of a multi-modality treatment that includes EBRT and/or chemotherapy and surgery. A summary of the clinical uses of HDR at various sites is also included this report.

\subsubsection{Carcinoma of the Cervix:}

In the curative treatment of cervical cancers, brachytherapy is a necessary component. Over the last decade, HDR has gained popularity in the U.S. due to the advantages alluded to earlier, specifically the possibility of therapy on an outpatient basis, avoidance of long-term bed rest, and avoidance of cervical dilation. Additionally, greater sparing of the rectum and bladder by temporary retraction, dose optimization, and integration with EBRT to the pelvis are possible. These advantages must be counterbalanced with the greater number of treatments required (typically five or six treatments lasting approximately 10-15 min each).

According to ABS recommendations, keep the total duration of treatment (EBRT and HDR) to less than 8 weeks, because prolongation adversely affects local control and survival .If HDR treatments (generally five or six fractions given once a week) were begun after completion of EBRT, the overall treatment duration would be unduly prolonged. The HDR is commonly given concurrently during the course of EBRT (but note that EBRT is not given on the day of HDR brachytherapy). The combined EBRT and HDR dose to point $A$ (or point $\mathrm{H}$ ) is an LDR equivalent of 80-85 Gy for early stage disease and 85-90 Gy for advanced stage [17], while non-bulky stage I/II less than $4 \mathrm{~cm}$ in diameter is defined as Early disease; and stage I/II greater than $4 \mathrm{~cm}$ in diameter or stage IIIB is defined as advanced diseased. The pelvic side-wall dose recommendations are 50-55 Gy for smaller lesions and 55-60 Gy for larger ones. The HDR dose is dependent on the stage of the disease and the dose of EBRT. Most centers use a schedule of approximately 6-8 Gy per fraction in four to six fractions, although two or three fractions of 8-10 Gy have been used (a smaller number of fractions is used by those using larger doses per fraction). [14], [15]

HDR doses can be obtained from the LDR equivalent using the linear-quadratic equation, as mentioned earlier. The ABS suggestions and the equivalent doses are given in Table (3.1) ${ }^{[16]}$ as a guide, recognize that many efficacious HDR fractionation schedules exist. The recommended HDR dose per fraction may vary by $\pm 0.25 \mathrm{~Gy}$. It is emphasized that extra care must be taken to 
ensure adequate bladder and rectal packing if high dose (>7 Gy) per fraction is used.

In certain difficult clinical situations (e.g., a narrow fibrotic vagina, bulky tumors, the inability to enter the cervical os, extension to the lateral parametria or pelvic side wall, lower vaginal extension and suboptimal applicator placement), the normal tissue tolerance may be exceeded if the above doses are used. In these situations, the HDR fraction size can be decreased (which requires an increase in the fraction number) or the EBRT dose increased while decreasing the HDR total dose, an interstitial implant (either LDR or HDR) may be used as an alternative.

LDR brachytherapy has been used with good results in carcinoma of the cervix for almost 100 years. Hence, it is important to critically analyze whether the results obtained with HDR brachytherapy, which has a much shorter history, compare with those obtained with LDR. Unfortunately, most of the published reports have been non-randomized studies. Although large, multi-institutional, randomized trials are not available, the available data from singleinstitution randomized trials, retrospective analyses and meta-analyses suggest that survival, local control, and morbidity of HDR treatments are equivalent to that of LDR. [18], [19], [20]

\subsubsection{Carcinoma of the Endometrium:}

In patients with intermediate and high risk for vaginal recurrence (high-grade, deep myometrial invasion, or advanced stage) after hysterectomy HDR brachytherapy is commonly used for adjuvant treatment of the vaginal cuff. Brachytherapy may be used for primary treatment in incurable endometrial carcinoma and for treatment of recurrences after hysterectomy.

Adjuvant radiation therapy should be used for the treatment of patients at high risk for vaginal recurrences after hysterectomy (deep myometrial invasion, high histological grade and stage, cervical or extra-uterine spread, squamous cell or papillary histology). Depending upon the extent of pelvic lymph node dissection Pelvic EBRT, vaginal vault brachytherapy or a combination can be used and whether chemotherapy will be added. It may be advantageous to use EBRT to irradiate the pelvic lymph nodes but takes approximately 5 weeks to perform and has some morbidity. It is more convenient to use brachytherapy and has low morbidity, but does not treat the lymph nodes. Some centers combined these two therapies but couldn't prove to produce superior results. Others prefer "watchful waiting" and use salvage irradiation. The dose is usually 40-45 Gy in 20-25 treatments, if pelvic EBRT is used. To deliver
HDR brachytherapy a vaginal cylinder is commonly used. To increase the depth dose the largest diameter cylinder that comfortably fits the vagina should be used. The length of vaginal vault treated varies. Some treat the superior $3 \mathrm{~cm}$ or $5 \mathrm{~cm}$, while others treat the superior half or two-thirds of the vagina. [21], [22]

Treatment of the entire vaginal canal should be considered for serious and clear cell histologies. Depending on the institutional policy, the dose distribution should be optimized to follow the curvature of the dome of the cylinder to deliver the prescribed dose either at the vaginal surface or at $0.5 \mathrm{~cm}$ depth. Doses to both of these points should be reported, regardless of the prescription point. ${ }^{[6]}$ The ABS dose suggestions for HDR alone or in combination with 45 Gy EBRT are given in Table 4.2. Since some institutions specify the dose to the vaginal surface and others specify the dose at $0.5 \mathrm{~cm}$ depth, suggested HDR doses have been given for both specification methods. Depending on the stage, grade, and depth of myometrial invasion, the 5-year survival rates of HDR therapy vary from $72 \%$ to $97 \%$. The severe (grades III or IV) late complication rate is usually less than $1 \%$ and depends on the dose per fraction. The incidence of vaginal shortening is also very much dose dependent, reportedly as high as $70 \%$ when 9 Gy per fraction was prescribed at $1 \mathrm{~cm}$ depth to $31 \%$ when the dose was reduced to 4.5 Gy per fraction. ${ }^{[23]}$ The use of a small $(2 \mathrm{~cm})$ diameter vaginal cylinder, the addition of pelvic EBRT and dose specification point beyond $0.5 \mathrm{~cm}$ are the other factors that increase the morbidity. ${ }^{[21]}$

To treat recurrences at the vaginal cuff a combination of pelvic EBRT and brachytherapy is generally used. For non-bulky recurrences (thickness less than $5 \mathrm{~mm}$ after the completion of EBRT) only Intracavitary vaginal brachytherapy should be used. For bulky recurrences (thickness $>5 \mathrm{~mm}$ after the completion of EBRT) Interstitial brachytherapy is to be used and for previously irradiated patients. The ABS suggested doses for HDR brachytherapy (in combination with 45 Gy EBRT) are provided in Table 4.3. ${ }^{[6]}$

Radiation therapy is used to treat the Patients with adenocarcinoma of the endometrium who are not candidates for surgery because of severe medical problems. Whenever possible a combination of pelvic EBRT beam and brachytherapy is preferred. However in these cases many of the conditions that do not allow surgery are also relative contraindications for EBRT and for LDR brachytherapy. These patients may be treated with HDR alone. For the treatment of primary endometrial cancer numerous applicators can be used. A tandem and colpostat is often used even if this applicator 
cannot irradiate the uterine surface homogeneously. Others have used a curved tandem, turning it to the left and right in alternate insertions. A "Y"-shaped applicator irradiates the fundus more evenly. Modified Heyman capsules or multiple tandems are other possibilities. Although computed tomography-based treatment planning to ensure a more homogeneous dose to the entire myometrium is preferred, the dose is commonly specified at $2.0 \mathrm{~cm}$. The dose per fraction has ranged from $5 \mathrm{~Gy}$ to $12 \mathrm{~Gy}$, and four to six fractions are commonly employed. [24], [25], ${ }^{[26]}$ If EBRT is added dose and/or then dose per fraction is reduced. The ABS-suggested doses for HDR brachytherapy alone or in combination with 45 Gy EBRT are given in Table 4.4. ${ }^{[6]}$ The survival at 5 years for stage-I is approximately $70-80 \%$, which is slightly lower than that obtained by surgery. When patients are treated with high doses per fraction, the toxicity is higher (about 7\%). ${ }^{[24]}$

Table 3.2. American Brachytherapy Society suggested doses of high-dose-rate (HDR) brachytherapy alone or in combination with external beam radiation therapy (EBRT) to be used for adjuvant treatment of post-operative endometrial cancer ${ }^{[6]}$

\begin{tabular}{|c|c|c|c|}
\hline \multicolumn{2}{|l|}{ EBRT (Gy) } & \multicolumn{2}{|l|}{ HDR dose } \\
\hline $\begin{array}{l}\text { @ } 1.8 \\
\text { Gy/fracti } \\
\text { on }\end{array}$ & $\begin{array}{l}\text { No. of } \\
\text { HDR } \\
\text { fractions }\end{array}$ & $\begin{array}{l}\text { per } \\
\text { fraction } \\
\text { (Gy) }\end{array}$ & $\begin{array}{l}\text { Dose } \\
\text { specification } \\
\text { poin }\end{array}$ \\
\hline 0 & 3 & 7 & $0.5 \mathrm{~cm}$ depth \\
\hline 0 & 4 & 5.5 & $0.5 \mathrm{~cm}$ depth \\
\hline 0 & 5 & 4.7 & $0.5 \mathrm{~cm}$ depth \\
\hline 0 & 3 & 10.5 & Vaginal surface \\
\hline 0 & 4 & 8.8 & Vaginal surface \\
\hline 0 & 5 & 7.5 & Vaginal surface \\
\hline 45 & 2 & 5.5 & $0.5 \mathrm{~cm}$ depth \\
\hline 45 & 3 & 4 & $0.5 \mathrm{~cm}$ depth \\
\hline 45 & 2 & 8 & Vaginal surface \\
\hline 45 & 3 & 6 & Vaginal surface \\
\hline
\end{tabular}

Table 3.3. American Brachytherapy Society suggested doses of high-dose-rate (HDR) brachytherapy to be used in combination with pelvic external beam radiation therapy (EBRT) for treating vaginal cuff recurrences from endometrial cancer ${ }^{[6]}$

\begin{tabular}{|c|c|c|c|c|}
\hline \multicolumn{2}{|c|}{ EBRT (Gy) } & \multicolumn{3}{|c|}{ HDR dose } \\
\hline $\begin{array}{l}\text { @ } 1.8 \\
\text { Gy/fracti } \\
\text { on }\end{array}$ & $\begin{array}{l}\text { No. of } \\
\text { HDR } \\
\text { fracti }\end{array}$ & $\begin{array}{l}\text { per } \\
\text { fraction } \\
\text { (Gy) }\end{array}$ & & $\begin{array}{l}\text { Dose } \\
\text { specification } \\
\text { point }\end{array}$ \\
\hline 45 & & 3 & & $0.5 \mathrm{~cm}$ depth \\
\hline 45 & & 4 & 6 & $0.5 \mathrm{~cm}$ depth \\
\hline 45 & & 5 & & Vaginal surface \\
\hline 45 & & 4 & 7 & Vaginal surface \\
\hline
\end{tabular}

Table 3.4. American Brachytherapy Society suggested doses of high-dose-rate (HDR) brachytherapy alone or in combination with external beam radiation therapy (EBRT) for treatment of inoperable primary endometrial cancer $^{[6]}$

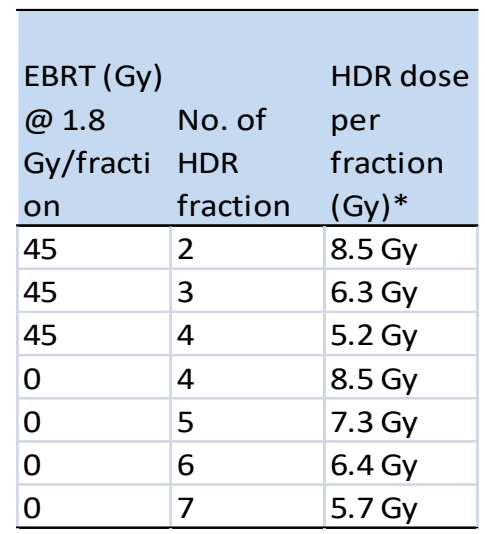

*HDR doses are specified at $2 \mathrm{~cm}$ from the midpoint of the intrauterine sources

\subsubsection{Endobronchial Radiation:}

For palliation of cough, dyspnea, pain, and hemoptysis in patients with advanced or metastatic lung cancer, the use of HDR brachytherapy is well established. In curative cases the use of brachytherapy as a boost to EBRT should be restricted to select a group of patients who have predominantly endobronchial disease, are medically inoperable, or have small/ occult carcinomas of the lung. To evaluate the airway and locate the site of obstruction an initial bronchoscopy is performed. Usually the proximal site of obstruction is visualized, while the distal extent of the obstruction may have to be estimated.

A 5- or 6-French catheter (inserted through the brush channel of the bronchoscope) can be used to deliver the brachytherapy. The catheter (with a radiopaque wire in the lumen) is passed through the obstructed segment and lodged into the distal bronchus. Leaving the catheter in position, the bronchoscope is removed. During bronchoscopic removal fluoroscopic guidance helps catheter positioning and minimizes inadvertent catheter dislodgment. The endobronchial tumor and $1.0-$ to $2.0-\mathrm{cm}$ proximal and distal margins are the lengths to be irradiated. It is possible to minimize the treatment planning time using preplanned dosimetry, if a single catheter is used and there is minimal curvature of the catheter in the area to be irradiated. For example, Ohio State University has pre-calculated treatment plans for 3-, 5-, 7-, and $10-\mathrm{cm}$ lengths to be irradiated to $5 \mathrm{~Gy}$ or 7.5 Gy at $1 \mathrm{~cm}$ from the source using equal dwell times. If standard lengths and doses are used then this allows the treatment to be performed without 
any delay. If multiple catheters are used, individualized image-based treatment planning must be performed.

Candidates for palliative endobronchial brachytherapy include: [27],[28], [29]

1. Symptoms such as shortness of breath, hemoptysis, persistent cough, and other signs of post-obstructive pneumonitis are produced by patients with a significant endobronchial tumor component. As opposed to extrinsic tumors that compress the bronchus or the trachea, tumors with a predominantly endobronchial component are considered suitable. Palliation of obstruction can be generally provided quickly by Endobronchial brachytherapy than EBRT. In addition to this, brachytherapy can be more convenient than 2-3 weeks of daily EBRT.

2. Patients who couldn't be able to tolerate any EBRT because of poor lung function.

3. Patients with previous EBRT of sufficient total dose to preclude further EBRT.

Various centers have used variety of doses successfully. A report has made on total doses ranging from 15 Gy to 47 Gy $\mathrm{HDR}$ in $1-5$ fractions calculated at $1.0 \mathrm{~cm} .{ }^{[30]}$. When HDR is used as the sole modality for palliation, the ABS suggests using three weekly fractions of $7.5 \mathrm{~Gy}$ each or 2 fractions of 10 Gy each or 4 fractions of $6 \mathrm{~Gy}$ each prescribed at $1.0 \mathrm{~cm}$. Using the linear quadratic model, similar radiobiological equivalence is of these fractionation regimes, and there is no evidence of superiority for one regime over the other.

Against the risks of a higher dose per fraction, the benefits of fewer bronchoscopic applications should be weighed.

For irradiated patients or those who have received limited radiation, additional treatments or doses higher than those suggested can be considered. The ABS suggests using 2 fractions of 7.5 Gy each or 3 fractions of 5 Gy each or 4 fractions of 4 Gy each (prescribed at $1.0 \mathrm{~cm}$ ) in patients with no previous history of thoracic irradiation, when HDR is used as a planned boost to supplement palliative EBRT of $30 \mathrm{~Gy}$ in 10 12 fractions. The interval between fractions is generally 1-2 weeks. When aggressive chemotherapy is given, the brachytherapy dose should be reduced. During brachytherapy, unless it is in the context of a clinical trial, concomitant chemotherapy should be avoided.

The results from various centers show bronchoscopy response from $59 \%$ to $100 \%$ and clinical improvement from $50 \%$ to $100 \% .{ }^{[31]}$ Due to the differences in patient populations and the variability in dose and fractionation employed, comparison of these results is difficult. Stenosis and Radiation bronchitis may occur after endobronchial brachytherapy, necessitating close follow-up.

Fatal hemoptysis is another more serious complication as it could be a radiation therapy complication resulting from the high dose delivered to the area of the pulmonary artery, or it could represent the failure of treatment due to the progression of disease. ${ }^{[32]}$ A high previous EBRT dose, a left upper lobe location, or longirradiated segments are multiple courses of brachytherapy, increase the rate of hemoptysis. $[33,34]$ Incidence of fatal hemoptysis varies from $0 \%$ to $50 \%$, with a median value of $8 \%$.

The definitive therapy for resectable lung cancer is a combination of chemotherapy and EBRT. As a boost to EBRT select patients with predominantly endobronchial tumor may benefit from endobronchial brachytherapy. [35, 36] Brachytherapy can be used to open up the bronchus and aerate the lung in cases of postobstructive pneumonia or lung collapse, which allows some sparing of normal lung from the EBRT field. Due to decreased pulmonary function, advanced age, or refusal of surgery, Endobronchial brachytherapy alone with curative intent is indicated in patients with occult carcinomas of the lung confined to the bronchus or trachea who are medically inoperable. [37],[ 38], [39] According to Marsiglia et al. [40], a survival rate of $78 \%$ with a median follow-up of 2 years in 34 patients treated with a HDR dose of $30 \mathrm{~Gy}$ in six fractions (5 Gy fractions given once a week). ${ }^{[40]}$

After surgical resection, Endobronchial brachytherapy can be used as adjuvant treatment in cases with minimal residual disease. Macha et al. ${ }^{[41]}$ reported tumor-free survival up to 4 years in 19 patients with doses of 20.0 Gy which is delivered in four fractions at $1 \mathrm{~cm}$ from the source axis. To boost to EBRT, the ABS recommends a HDR dose of three 5-Gy fractions or two 7.5-Gy fractions, either $60 \mathrm{~Gy}$ in 30 fractions or $45 \mathrm{~Gy}$ in 15 fractions. The HDR dose should be prescribed at a distance of $1.0 \mathrm{~cm}$ from the central axis of the catheter and given weekly. In previously unirradiated patients, HDR doses of five 5-Gy fractions or three 7.5-Gy fractions prescribed to $1 \mathrm{~cm}$ may be used If endobronchial brachytherapy is used alone. ${ }^{[31]}$

\subsubsection{Cancer of the Esophagus:}

Treatment for advanced cancer of the esophagusis essentially palliative but the results are dismal (5-year survival $=6 \%$ ). HDR brachytherapy can be used either in combination with EBRTor alone in the treatment of esophageal cancer. ${ }^{[42]}$ As a single catheter is 
used for the treatment so Brachytherapy is relatively simple to perform. A specially designed esophageal applicator or a nasogastric tubeis used to deliver the treatments. The largest diameter applicator, inserted easily either intraorally or intranasally, should be used to minimize the mucosal dose relative to the dose at depth. The site to be irradiated includes the tumor and a margin of $2-5 \mathrm{~cm}$ can be confirmed by endoscopy or fluoroscopy. The ABS suggests a HDR dose of 10 Gy in two fractions, to boost 50Gy EBRT, prescribed at $1 \mathrm{~cm}$ from the source. HDR brachytherapy can be given before, concurrent with, or after EBRT. A more uniform dose can be delivered to the residual tumorafter EBRTafter it has been reduced using EBRT is the advantage of giving brachytherapy. Brachytherapy given initially provides rapid relief of dysphagia. At doses of $16 \mathrm{~Gy}$ in two treatments, HDR brachytherapy have been used without additional EBRT to palliate esophageal cancers. ${ }^{[43]}$

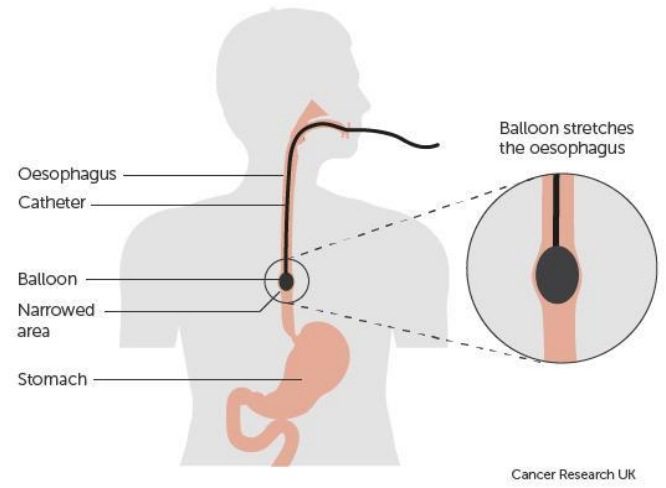

Figure 3.1: Internal radiotherapy with radioactive source through a NGT tube ${ }^{[45]}$

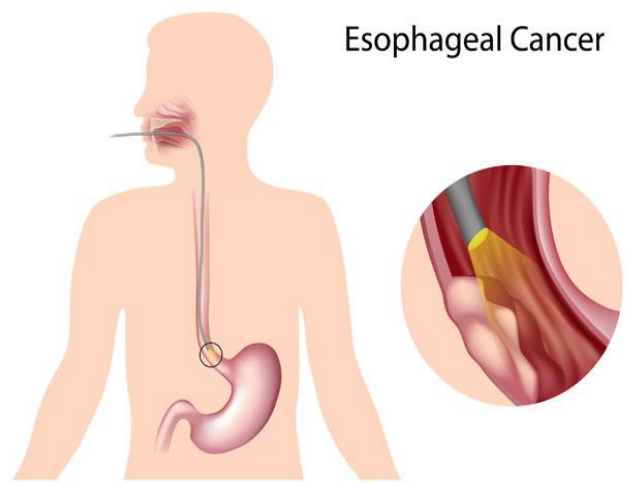

Figure 3.2: narrowing of food pipe ${ }^{[45]}$

Retrospective studies as well as prospective, randomized clinical trials show that there is improved local control and survival when HDR brachytherapy is added to EBRT. HDR brachytherapy alone can be used for palliation of advanced esophageal cancers. ${ }^{[4]}$ The side effects of a high dose delivered to the esophageal mucosa may include ulcerations, fistulae, and esophageal strictures.

\subsubsection{Carcinoma of the Prostate:}

Currently, the most common type of prostate brachytherapy is permanent implantation of palladium-103 seeds or iodine-125. However, several centers have used HDR brachytherapy for the treatment of prostate cancer, usually as a

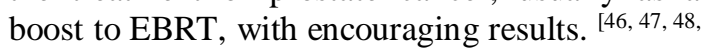
${ }^{49,50]}$ HDR has a major advantage is that the dose distribution can be intraoperatively optimized by varying the dwell times at various dwell positions, ${ }^{[51]}$ potentially allowing reliable and reproducible delivery of the prescribed dose to the target volume while keeping the doses to normal structures, i.e., bladder, urethra, and rectum, within acceptable limits. The theoretical consideration, another potential advantage of HDR brachytherapy in prostate cancer is that prostate cancer cells behave more like latereacting tissue with a low alpha-beta ratio and they should, therefore, respond more favorably to higher dose fractions rather than to the lower dose rate delivered in LDR brachytherapy. ${ }^{[52,53]}$ Standard fractionation EBRT (39.6-50.4 Gy) is given concurrent with or within 2 weeks before or after HDR brachytherapy. The minimum volume treated should include the whole prostate and seminal vesicles with margin, with or without pelvic lymph nodes. The HDR dose is given in multiple fractions in one or two implant procedures.

\section{Healthy}

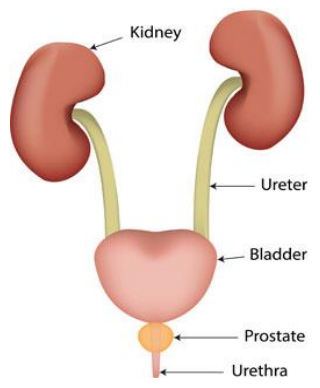

\section{Prostate Cancer}

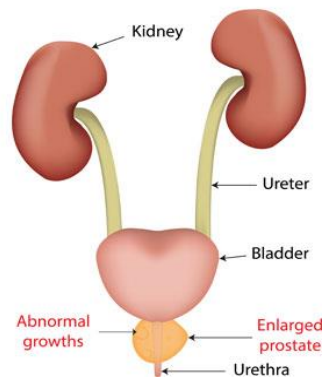

Figure 3.3: Prostate Cancer Diagnosis ${ }^{[54]}$

A variety of dose and fractionation schemes have been used for same-stage disease as shown in Table $3.5^{[55]}$. The HDR fractions are generally given two times in a day with a minimum of $6 \mathrm{~h}$ between fractions. The most commonly encountered acute genito-urinary morbidities include urinary irritative symptoms, hematospermia, hematuria, and/or urinary 
retention, similar to LDR permanent implants. In a few centers HDR brachytherapy has also been used as monotherapy, but long period results are awaited. HDR doses of 38 Gy delivered in four fractions, two times daily for 2 days or $54 \mathrm{~Gy}$ in nine fractions given two times in a day over 5 days have been reported. ${ }^{[56],[57]}$

Table 3.5. Equivalent doses and dose fractionation (as if given at 2 Gy per fraction) of common combined high-dose-rate (HDR) and external beam radiation therapy (EBRT) doses used for treating prostate cancer ${ }^{[55]}$

\begin{tabular}{|c|c|c|c|c|c|c|}
\hline $\begin{array}{l}\text { EBRT } \\
\text { dose (Gy) }\end{array}$ & $\begin{array}{l}\text { Total } \\
\text { HDR dose } \\
\text { (Gy) }\end{array}$ & $\begin{array}{l}\text { HDR } \\
\text { dose/ } \\
\text { fraction } \\
\text { (Gy) }\end{array}$ & $\begin{array}{l}\text { No. of } \\
\text { HDR } \\
\text { fractions }\end{array}$ & $\begin{array}{l}\text { Equivale } \\
\mathrm{nt} \text { dose } \\
(\mathrm{Gy})^{*} \\
(\alpha / \beta=1.5)\end{array}$ & $\begin{array}{l}\text { Equivale } \\
\text { nt dose } \\
(G y)^{*} \\
(\alpha / \beta=5)\end{array}$ & $\begin{array}{l}\text { Equivale } \\
\text { nt dose } \\
(G y)^{*} \\
(\alpha / \beta=10)\end{array}$ \\
\hline 39.6 & 22 & 5.5 & 4 & 81 & 72 & 67 \\
\hline 45 & 18 & 6 & 3 & 81 & 72 & 68 \\
\hline 39.6 & 26 & 6.5 & 4 & 97 & 81 & 75 \\
\hline 50.4 & 19.5 & 6.5 & 3 & 92 & 81 & 72 \\
\hline 46 & 19 & 9.5 & 2 & 106 & 85 & 77 \\
\hline
\end{tabular}

Prostate cancer is a health issue not only for men in Western countries but also for men worldwide, especially considering recent epidemiological trends. The total number of diagnosed prostate cancer cases has increased, with the introduction of the prostate specific antigen (PSA) test. Recently, the results of a large European trial which was related to PSA screening showed a reduction in prostate cancer associated mortality. ${ }^{[58]}$ However, a trial in the United States of America addressing the same subject report dissimilar results. ${ }^{[59]}$ The most important adverse consequences of prostate cancer screening are overtreatment and over diagnosis. For all men over the age of 75 years, the United States Preventive Services Task Force has recommended PSA screening. [60] In consideration of higher cure rates with similar or lower incidences of late effects compared with standard radiation therapy, dose escalation above 70 Gy has been proven to be beneficial in prostate cancer radiotherapy. Dose escalation can be performed either with EBRT techniques (i.e. proton beams, 3-D conformal radiation therapy and IMRT) or with BT using 103Pd seed implants or temporary $192 \mathrm{Ir}$ or permanent $125 \mathrm{I}$. [61], [62] Because the risk group affects the outcome dramatically, therefore the current standard of practice is to stratify patients according to predictive factors into risk groups. Patients who have localized prostate cancer are usually divided into three risk groups: low, intermediate and high, for practical purposes. Although there are many schemes that are proposed, In the United States of America, National Comprehensive Cancer Network guidelines have been the most widely adopted (see Table 3.6). ${ }^{[63]}$

Table 3.6. National Comprehensive Cancer Network Risk Stratificaton ${ }^{[63]}$

\begin{tabular}{l|l|}
\hline Risk level & Criteria \\
\hline Low & T1-T2a and Gleason score $2-6$ and PSA $<10 \mathrm{ng} / \mathrm{mL}$ \\
\hline Intermediatea & T2b-T2c or Gleason score 7 or PSA $=10-20 \mathrm{ng} / \mathrm{mL}$ \\
\hline Higha & T3a or Gleason score $8-10$ or PSA $>20 \mathrm{ng} / \mathrm{mL}$ \\
\hline Locally advanced very high & T3b-T4 \\
\hline $\begin{array}{l}\text { Metastatic } \\
\text { Any T N1 or any T, any N with M1 }\end{array}$ \\
\hline Patients with multiple adverse factors can be shifted into the next higher risk group.
\end{tabular}

The optimal management of both localized and locally advanced prostate cancer remains controversial. To treat the different risk groups, radiotherapy, Surgery and hormonal therapy can be used alone or in combination. ${ }^{[64]}$

Theoretical advantages of BT in the form of temporary implantations include:

1) Potential for 'boost-in-boost' radiation due to steep dose fall-off in the peripheral zone, which is the most common target location.

2) A shorter learning curve compared with LDR implantations.

3) No movement of the source in relation to the target volume within the time interval of radiation.

4) More effective dose volume optimization potential of the stepping source technology. 
5) Potential of less toxicity due to the improved protection of risk areas, such as the bladder base as well as the penile bulb, urethra and rectum.

There are some disadvantages compared with the seed implant approach discussed in the literature:

1. A high level of quality assurance is necessary before each application of radiation, because fractionation can occur with one implant, the needle to target relationship can change compared with the initial situation.

2. On quality of life, lack of prospective and comparative randomized studies.

3. Unclear cost compared with LDR treatments. However, HDR monotherapy could have an economic advantage, in high workload centres.

\section{a) High dose rate brachytherapy as a boost}

Use as a local dose escalation method that is complementary to external beam techniques, is the most common application of temporary BT in prostate cancer. This combination allows a very high local dose to the tumour and lower normal tissue doses in the surrounding tissue. This treatment is indicated in intermediate or high risk cases that do not have metastases or nodal involvement commonly used dose schedules available in the literature are contraindications and indications. [65] Doses and fractions are widely variable, but they are in the range 1-15 Gy to four fractions of $3 \mathrm{~Gy}$.

Long term follow-up data confirm that the HDR boost combined with external beam radiation results in excellent biochemical control rates. [66], [67] In experienced hands, a 67-78\% biochemical relapse free rate is achievable with a genitourinary/gastrointestinal toxicity rate of 5$7 \%$ for greater than $\mathrm{G} 3$ complications. Usually, approximately $30-40 \%$, temporary BT causes the same level of erectile dysfunction as permanent or LDR seed implants. Urethral strictures (approximately 8\% G2 or higher) are the most common treatment related injuries following HDR prostate BT. Both dosimetry and clinical factors appear to influence the risk of stricture formation. [68], [69]

It is noteworthy that androgen deprivation has a role in conjunction with BT. The role of combining neoadjuvant androgen deprivation and permanent prostate BT was to reduce the prostate to a size suitable for optimal implantation. Ebara et al. ${ }^{[70]}$ show that a three month course of the neoadjuvant luteinizing hormone releasing hormone agonist resulted in effective volume reduction of $32-35 \%$ for an enlarged prostate.

Temporary BT using stepping source technology does not require special source preparation and causes no post-implant radiation protection problems. It also allows fractionated treatment schedules as well as individual dose optimization and high delivery quality assurance. The only disadvantage compared with LDR permanent seed implants is the need for fractionation, which results in a higher workload for the department. On the other hand, there might be some cost benefits to HDR implants, which can be seen if one compares the costs of the LDR seeds (which are used once per patient) and the HDR treatment (which is given over a fixed time period). The costs of the radiation source and workforce in the HDR treatments are stable, while the growing number of implanted seeds purchased for each patient continues to add up. This benefit would be applicable in departments that have a high volume of implants.

\section{b) High dose rate brachytherapy as monotherapy}

HDR fractionated monotherapy for prostate cancer was introduced by Yoshioka et al. ${ }^{[71]}$, and feasibility studies were published by Martinez et al. ${ }^{[72]}$ and Martin et al. ${ }^{[73]}$. Standard fractionated EBRT (i.e. 3-D EBRT and IMRT) or permanent seed implantation are the most common methods for delivering radiotherapy in low risk patients. However, radiobiological considerations (very low $\alpha / \beta$ ratio of prostate cancer) suggest that hypofractionation could be an advantage due to much shorter treatment times and lower costs than those in IMRT and seed implants, with comparable outcome levels. ${ }^{[74],[75]}$

HDR BT as monotherapy has indications that are very similar to those used for seed implantation, for patients with low risk prostate cancer. There is a difference in the fractionation approach: some groups use three to four implantations, while others use two implantations with three to four fractions. The dose per fraction varies in the range of 8.0-9.6 Gy in the peripheral zone. The time interval between the fractions needs to be a minimum of six hours. There are no published phase III clinical investigations that compare HDR monotherapy with other radiotherapy methods. The available phase II studies suggest that there is an excellent biochemical response with no differences seen in acute and late toxicity between the dose schemes of $34 \mathrm{~Gy}$ in four fractions, $36 \mathrm{~Gy}$ in four fractions or $31.5 \mathrm{~Gy}$ in three fractions. [76], [77]

Experienced groups report five year biochemical control rates of $91 \%$ (Phoenix 
definition, nadir $+2 \mathrm{ng} / \mathrm{mL}$ ) and local control rates of $98.9 \%$. ${ }^{[78]}$

The incidence of BT relating to normal tissue injury could be minimized by using advanced technology or strict rectal dose constraints (V40 $<8 \mathrm{~cm} 3$ and D5 $\mathrm{cc}>27 \mathrm{~Gy}$ ) as well as by keeping the volume of high dose areas low. [79], [80] Rigorous quality assurance practices can avoid unplanned dosimetry changes between different fractions by using the same implant. ${ }^{[81]}$ The role of antiandrogen treatment as a complementary approach to HDR monotherapy in prostate cancer has not yet been systematically investigated.

HDR fractionated monotherapy is still in its early development. The relatively small number of reported patient series and the relatively short follow-ups require regular updates to produce mature data. Therefore, evidence based consensus is required for generating dose and fractionation schedule guidelines for this type of prostate interstitial BT.

\section{c) High dose rate brachytherapy as salvage treatment}

HDR BT has been used as a salvage approach for isolated local recurrences after previous EBRT or permanent seed implantation. [82], [83]

\section{d) High dose rate prostate implantation technique}

HDR implantation can be performed before or after EBRT because an insignificant prostate volume change is expected. [84] However, individual variations can occur, and the effect of oedema should be considered when planning EBRT after single fraction HDR prostate BT. ${ }^{[85]}$ In most cases, HDR implantation is performed under spinal or general anaesthesia with the patient in the lithotomy position. Data on the use of local anaesthesia with or without sedation is also available. [86], [87] Different perineal templates can be used for needle guidance to obtain an optimal implant (see Figure 3.4).
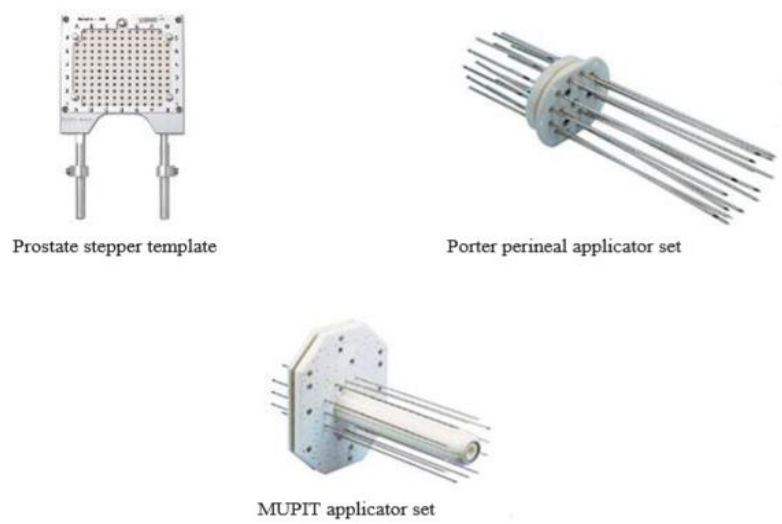

Note: MUPIT - Martinez universal perineal interstitial template.

Figure.3.4: Applicators for prostate HDR BT (courtesy of Nucletron, Netherlands).

A transrectal ultrasound (TRUS) probe that is linked to a stepping unit is usually positioned as parallel as possible to the prostatic urethra. The apex and base of the gland need to be clearly identified. Fiducial markers are inserted into the apex and the base of the prostate to ensure quality control of any needle displacement during the treatment and to allow corrections whenever necessary. ${ }^{[88]}$

Since real time TRUS based procedures can provide better prostate imaging quality than $\mathrm{CT}$, and the procedure can be performed in the operating room within 10-15 minutes, this method is recommended in many existing guidelines. Descriptions of both the TRUS based and CT based procedures are given below, and the steps of a TRUS based HDR implant procedure are detailed in Ref. [89] following adequate anaesthesia and positioning of the patient in the lithotomy position, a Foley catheter is introduced. The catheter can be filled with aerated gel for improved visibility on TRUS images.

After checking for potential pubic arch interference, as well as for prostate position projection to the perineal template grid on the screen of the TRUS machine, a 3-D TRUS volume is created. Imaging starts at half of the Foley balloon in the bladder and finishes at the penile bulb. Additional methods can help to define the geographical location of the intraprostatic tumour load (e.g. magnetic resonance spectroscopy image matching and Doppler TRUS) and can influence the planned 
needle geometry within the prostate. Needles are implanted from the medial to the lateral section of the gland using axial TRUS image guidance.

The peripheral zone and detectable areas of capsule invasion are usually implanted with approximately $1 \mathrm{~cm}$ needle separation. If necessary, additional needles are used to cover the apical part of the prostate. In the case of base involvement, needle tips are inserted into the bladder because the first possible source position is approximately $6-8 \mathrm{~mm}$ behind the trocar tip of the needle. The implantation starts in the ventrodorsal direction, and right/left needles are implanted, one after the other, to avoid procedure related torsion of the gland. On the sagittal view, each needle is then forwarded to the base of the prostate under visual control.

After completing the implant procedure, a 3D TRUS volume is created and analysed to control the needle geometry. It is easy to improve its geometry, if necessary. If the geometry of the implantation is acceptable, then the capture of 1.0 $\mathrm{mm}$ transverse images via a video connection from the TRUS unit to the planning computer is performed. The capture starts at least $5.0 \mathrm{~mm}$ cranial to the needle tips and ends $5.0 \mathrm{~mm}$ caudal from the apex. Delineation of the volumes of interest (prostate, rectum, bladder and urethra) is performed, and individual needle positions in the virtual 3-D volume are noted.

After creating an appropriate dose distribution, the needles are connected to the afterloading machine and radiation is given. After completing a fraction of the radiation treatment, the needles and any in vivo dosimetry devices are removed. Recommended dose constraints vary between different publications and are listed in the GEC-ESTRO temporary BT recommendations. ${ }^{[65]}$

Alternatively, Slessinger et al. ${ }^{[84]}$ describe the CT based HDR BT technique. TRUS is used to identify the prostate and to place gold marker seeds at the base and apex. With the stepper stabilizer and template in place, needle placement is performed at the largest cross-section of the prostate. Needles are placed to allow for peripheral coverage with approximately $1 \mathrm{~cm}$ needle spacing. In addition, two to four interior needles, depending on the prostate size, are placed midway between the urethra and the peripheral needles. Fluoroscopy and flexible cystoscopy are used to confirm adequate needle insertion depth. Once the needle implantation is completed, a template photograph is obtained in the operating room. A special CT compatible board can be used to move the patient from the operating room table to the CT table and to the hospital bed because a stable needle insertion depth requires leg movement to be minimized. CT scanning is performed once the patient is released from the recovery room. CT images (with diluted contrast filling the bladder) are obtained to evaluate and adjust the needle insertion depth to assure adequate coverage at the prostate base. Once the adjustments are complete, rectal contrast is introduced, the needle obturators are withdrawn and $2.5 \mathrm{~mm}$ axial CT slices are acquired from the level of the midFoley balloon to the perineum. The needles contain only air and appear as dark spots on the CT images.

After the CT scan, the level at which the needles emerge from the template are marked to document the catheter position. The CT study is exported to the treatment planning computer. The radiation oncologist delineates the planning target, urethra and rectal dose points. Implant needle catheters are then reconstructed, and active dwell positions are selected. The maximum urethral dose is limited to $110 \%$ of the prescription dose based on the contoured volume, and the anterior rectal dose points at the rectal contrast interface are not allowed to exceed $75 \%$. In addition, the volume that receives $125 \%$ and $150 \%$ of the prescribed dose should not be greater than $50 \%$ and $25 \%$ of the target volume, respectively. The total planned treatment time is verified using an independent method. A range of doses has been deemed to be acceptable. Slessinger et al. ${ }^{[84]}$ recommend 9.5 Gy followed seven days later by another implant that delivers another 9.5 Gy when the BT is administered as a boost to supplement the external beam radiation. The patients who are treated with monotherapy receive six HDR treatment fractions (7 Gy/fraction). For monotherapy, the patient has two operating room procedures, each of which is associated with an operating room day treatment, and two fractions the following day, which are a minimum of six hours apart. The following day, before the treatments, radiographic imaging is obtained. Adjustments to the catheter insertion depth are made based on a comparison with the baseline orthogonal film set obtained shortly after the planning CT scan.

It is important to note that on completion of each treatment session, the patient is surveyed using a calibrated radiation instrument to confirm that the HDR source is safely stored.

\section{A summary of the HDR implantation procedure is given in the following:}

(a) A perineal template that will be used for needle guidance during the procedure is prepared.

(b) Following adequate anaesthesia and positioning of the patient in the lithotomy position, a Foley catheter filled with aerated gel is introduced. 
(c) To create a 3-D TRUS volume, imaging for the volume starts at the half of the Foley balloon that is in the bladder and finishes at the bulb of the penis. Implantation of needles with approximately $1 \mathrm{~cm}$ of needle separation is performed from the medial to the lateral section of the gland, using axial TRUS image guidance. The implantation starts in the ventrodorsal direction, and right/left needles are implanted. On the sagittal view, each needle is then forwarded to the base of the prostate under visual control.

(d) Fluoroscopy and flexible cystoscopy are used to confirm an adequate needle insertion depth.

(e) Once the needle implantation is completed, a template photograph is obtained in the operating room. Imaging is performed after the procedure. The type of imaging depends on the BT technique: If the BT technique is ultrasound based, then a 3-D TRUS volume is created after the procedure. Corrections to the geometry can be performed at this point. If the BT technique is CT based, then a CT compatible board is used to move the patient from the operating room table to the CT table and to the hospital bed to maintain a stable needle insertion depth. CT scanning is performed, and adjustments can be made to the needle depth.

(f) The volumes of interest (prostate, rectum, bladder and urethra) are delineated. Implant needle catheters are then reconstructed, and active dwell positions are selected. Planning is performed based on $1 \mathrm{~mm}$ transverse images.

(g) Before each fraction, radiographic imaging is obtained. Adjustments to the catheter insertion depth are performed based on a comparison with the baseline orthogonal film set obtained shortly after the planning CT scan. Fiducial markers should be placed at the apex and base if this method of verification is used.

(h) The needles are connected to the afterloading machine, and radiation is administered. After completing the radiation treatment fraction, the needles and any in vivo dosimetry devices are removed.

(i) It is important to note that on completion of each treatment session, the patient is surveyed using a calibrated radiation instrument to confirm that the HDR source is safely stored.

\subsubsection{Head and Neck Cancers:}

Brachytherapy, especially using manually afterloaded iridium-192, has been widely used to treat head and neck cancers. HDR brachytherapy has been used in selected cases to reduce radiation exposure and permit optimization as summarized in Table 3.7 [90], [91], [92], [93], [94], [95]. However, these advantages are offset by the need for multiple fractionations, since the head and neck area does not tolerate high doses per fraction.

The nasopharynx is a site within the head and neck area that is easily accessed by an intracavitary HDR applicator. ${ }^{[96]}$ Doses of $18 \mathrm{~Gy}$ in six fractions are delivered by a special nasopharynx applicator to boost 46-60 Gy of EBRT. ["7]

The use of HDR brachytherapy catheters incorporated in removable dental molds allows repeated, highly reproducible, fractionated outpatient brachytherapy of superficial (less than $0.5-\mathrm{cm}$ thick) tumors without requiring repeated catheter insertion into the tumor. ${ }^{[98]}$ Suitable sites for mold therapy include the scalp, face, pinna, lip, buccal mucosa, maxillary antrum, hard palate, oral cavity, external auditory canal, and the orbital cavity after exenteration. HDR can be used as the sole modality or in conjunction with EBRT. A total HDR dose equivalent to approximately 60 Gy LDR (prescribed at $0.5-\mathrm{cm}$ depth) is recommended when used as the sole modality. ${ }^{[99]}$ The HDR can also be used as a boost to 45-50 Gy EBRT, in which case, the HDR doses are appropriately reduced to LDR equivalent doses of 15-30 Gy. The actual HDR dose per fraction and number of fractions can be varied to suit individual situations (including site and treatment volume). Biomathematical (linearquadratic) modeling can be used to assist in the conversion of LDR to HDR ${ }^{[100]}$

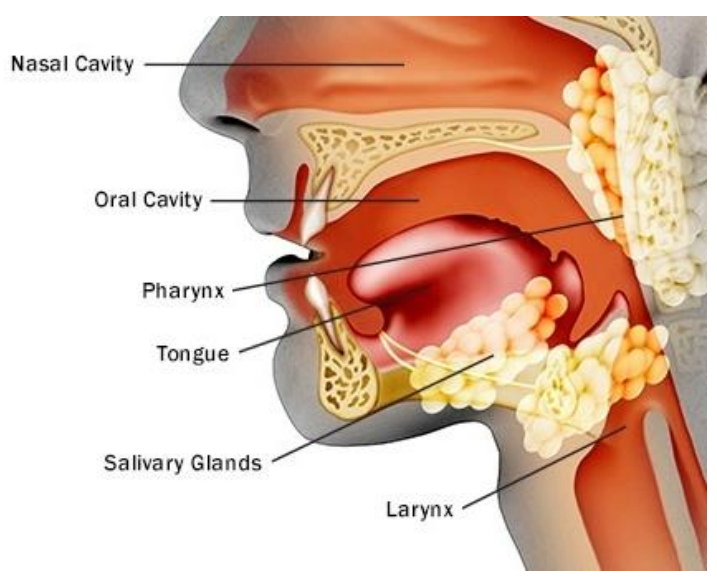

Figure 3.7: Where do they start ${ }^{[101]}$ 
Table 3.7. High-dose-rate (HDR) brachytherapy for head and neck cancers. LC (local control), EBRT (external beam radiation therapy) ${ }^{[90,91]}$

\begin{tabular}{|c|c|c|c|c|c|c|}
\hline Author & $\begin{array}{l}\text { EBRT } \\
\text { dose (Gy) }\end{array}$ & $\begin{array}{l}\text { Fraction } \\
\text { size (Gy) }\end{array}$ & $\begin{array}{l}\text { No. } \\
\text { fractions }\end{array}$ & $\begin{array}{l}\text { Equivale } \\
\text { nt dose* } \\
\text { (Gy) }\end{array}$ & $\begin{array}{l}\text { No. of } \\
\text { patients }\end{array}$ & $\mathrm{LC}$ \\
\hline Dixit et al. 1997 & 0 & 3 & 20 & 65 & 3 & - \\
\hline Lau et al. 1996 & 0 & 6.5 & 7 & 63 & 27 & $53 \%$ \\
\hline Inoue et al. 1996 & 0 & 6 & 10 & 80 & 14 & $100 \%$ \\
\hline Donath et al. 1995 & 0 & $4.5-5$ & 10 & $54-63$ & 13 & $90 \%$ \\
\hline Leung et al. 1998 & 0 & $5.5-6$ & 10 & $71-80$ & 13 & $100 \%$ \\
\hline Yu et al. 1996 & 50 & 2.7 & 6 & 67 & 12 & $79 \%$ \\
\hline Dixit et al 1997 & $40-48$ & 3 & 7 & $63-71$ & 18 & $80 \%$ \\
\hline
\end{tabular}

*Equivalent dose for tumor effects as if given at $2 \mathrm{~Gy} /$ day using the linear quadratic model with an $\alpha / \beta$ ratio of $10^{[100]}$

Another innovative approach is the use of intraoperative HDR brachytherapy, which permits normal tissues to be retracted or shielded during brachytherapy. Intraoperative HDR brachytherapy can reach many sites in the head and neck area that are difficult to treat or are inaccessible by either LDR brachytherapy or intraoperative electron beam radiation. The catheters are removed immediately after the single dose of radiation, hence, minimizing inconvenience and permitting the use of brachytherapy in areas such as the base of skull. ${ }^{[102]}$ Doses of 7.5-15.0 Gy are given when EBRT of 45-50 Gy can be added. In recurrent tumors where no further EBRT can be given, a single intraoperative dose of 15-20 Gy can be given.

\subsubsection{Soft Tissue Sarcomas:}

Excellent results are obtained with a combination of wide excision of the tumor and adjuvant EBRT. However, irradiation of large volumes after surgery gives rise to morbidity, especially normal tissue fibrosis. A few centers have used LDR brachytherapy to minimize morbidity and improve local control. [103], [104] The major problem with LDR brachytherapy of large volumes is the radiation exposure involved. Hence, a few centers are investigating the use of HDR brachytherapy for soft tissue sarcomas. [105], [106], [107] HDR brachytherapy catheters are implanted approximately $1 \mathrm{~cm}$ apart along the tumor bed, and radio-opaque clips indicate the margins. A 2- to 5-cm margin proximally and distally is used after gross excision of tumor. Optimized treatment planning can be used to deliver a more homogeneous dose. Doses of 4050 Gy are given in 12-15 fractions if the HDR is given alone. If EBRT (45-50 Gy) is added, the brachytherapy dose is limited to $18-25$ Gy in 4-7 fractions. An alternative technique not widely available is intraoperative HDR brachytherapy (HDR-IORT). ${ }^{[108]}$ A HDR-IORT dose of 12-15 $\mathrm{Gy}$ is given to the tumor bed in a single fraction intraoperatively to boost EBRT doses of 45-50 Gy. Nerve tolerance to high dose per fraction is poor, and HDR should be used with caution when catheters have to be placed in contact with neurovascular structures.

The ABS suggests the following interventions to minimize morbidity in soft tissue sarcomas [109]:

1. When brachytherapy is used as adjuvant monotherapy, the source loading should start no sooner than 5-6 days after wound closure. However, the radioactive sources may be loaded earlier (as soon as 2-3 days after surgery) if doses of less than 20 Gy are given with brachytherapy as a supplement to EBRT.

2. Minimize dose to normal tissues (e.g., gonads, breasts, thyroid, skin) whenever possible, especially in children and patients of childbearing age.

3. Limit the allowable skin dose - the 40-Gy isodose line (LDR) to less than $25 \mathrm{~cm} 2$ and the 25-Gy isodose line to less than $100 \mathrm{Cm}^{2}$.

\subsubsection{Pediatric Tumors:}

LDR brachytherapy has been used in children to reduce the deleterious effects of EBRT. [110] However, LDR brachytherapy is difficult to perform in young children and infants because they require prolonged sedation and immobilization with close monitoring, which increases the risk of radiation exposure to nursing staff and parents. HDR is, therefore, very appealing for infants and younger children and is currently undergoing trials at Ohio State University. [111], [112] The recommended dose for HDR as monotherapy is 36 Gy in 12 fractions given at $3 \mathrm{~Gy}$ (prescribed at $0.5 \mathrm{~cm}$ ) twice a day. The interval between fractions is at least $6 \mathrm{~h}$. There are no published dose recommendations for HDR as a boost to EBRT. The linearquadratic model can be used to calculate a 
fractionation scheme equivalent to that of a LDR implant boost-dose of 15-25 Gy (prescribed at $0.5 \mathrm{~cm})$. The recommended dose for intraoperative HDR brachytherapy is $10-15$ Gy (prescribed at $0.5 \mathrm{~cm}$ ), as a boost to 30-40 Gy EBRT, depending on the extent of residual disease. [113], [114] Although the long-term morbidity of HDR brachytherapy in young children is not fully known, one may expect preservation of organ function similar to that seen with LDR brachytherapy. Due to the complexities involved in pediatric HDR brachytherapy, it is recommended that the use of HDR brachytherapy in pediatric tumors be limited to centers that have experience with pediatric implants. ${ }^{[15]}$

\subsubsection{Breast Cancer:}

\section{Brachytherapy for Early-Stage Breast Cancer;}

\section{A type of radiation therapy that treats cancer} at its source

Brachytherapy, also referred to as internal radiation therapy, is a form of cancer treatment in which a sealed radioactive source is placed in or near a tumor to kill it. It is used to treat many different types of cancer, but is specifically used in breast cancer to clear cancer cells following a lumpectomy. Brachytherapy may also be used to reduce the size of a tumor prior to breast cancer surgery or as palliative treatment to reduce pain or bleeding in advanced or inoperable disease. ${ }^{[116]}$

Breast brachytherapy is used to treat earlystage cancers that have not spread (metastasized) to other parts of the body. The treatment can be delivered in different ways and is often combined with conventional external beam radiation therapy (EBRT).

\section{Purpose of Treatment:}

EBRT is the standard of care for women who have undergone a lumpectomy or mastectomy. Breast brachytherapy can help support EBRT by "boosting" the response rate and improving outcomes. [117] In recent years, high-dose brachytherapy has been used as a standalone treatment of early-stage cancer, a procedure referred to as accelerated partial breast irradiation (ABPI). Brachytherapy is also sometimes used in neoadjuvant therapy, a form of treatment designed to shrink a tumor before surgery. Doing so can reduce the risk of cancer recurrence by decreasing the tumor margins (the transitional areas between normal and cancerous tissue), where cancer cells hide. ${ }^{[118]}$ One of the main advantages of breast brachytherapy compared to whole-breast irradiation is that a higher dose of radiation can be precisely delivered with less damage to surrounding tissues of the breast, ribs, and lungs.

\section{Brachytherapy Types for Breast Cancer:}

Both the implants and implantation methods used in brachytherapy can vary by the cancer stage and type. The implants may be temporary or permanent and vary in size and radioactive strength. Depending on the procedure, the specialist (known as a radiation oncologist) may use radioactive seeds, pellets, capsules, ribbons, tubes, or balloons.

There are five methods used to deliver breast brachytherapy, each of which has specific purposes and indications. ${ }^{[118]}$

1. Interstitial breast brachytherapy involves the placement of several flexible tubes (catheters) in the breast tissue through which a radiation source is placed.

2. Intracavity breast brachytherapy, also known as balloon brachytherapy, is used after a lumpectomy to deliver radiation to the breast cavity via an inflatable balloon.

3. Intraoperative radiation therapy delivers localized radiation during the lumpectomy via a cone-shaped applicator and a radiation emitter.

4. Permanent breast seed implant (PBSI) involves the permanent implantation of low-dose radioactive seeds to prevent cancer recurrence in women with early-stage breast cancer.

5. Non-invasive breast brachytherapy (NIBB) is a newer procedure that doesn't require catheters or implantation but instead delivers a concentrated beam of radiation while the breast is compressed during a mammogram.

According to a 2017 review of studies, [119] breast brachytherapy can reduce the rate of cancer recurrence from 13.3 percent to 6.3 percent when used with EBRT.

\section{Dosage:}

The type of radioactive material used in brachytherapy (typically iodine, palladium, cesium, or iridium) varies by the treatment type. The material, called the source, is encapsulated in a metal shell that controls how much radiation is emitted.

The length of time an implant is left in place depends on the type of brachytherapy used. This is directed by the dose rate, described in units called Grays per hour (Gy/hr). With breast cancer, there are five possible dose rates. ${ }^{[18]}$ 
- Ultra-low-dose rate (ULDR)

brachytherapy involves seeds or pellets that emit radiation at 0.1 to $0.3 \mathrm{~Gy} / \mathrm{hr}$.

- Low-dose rate (LDR) brachytherapy involves implants that emit radiation at 2 Gy/hr.

- Medium-dose rate (MDR) brachytherapy emits radiation at 2 to 12 Gy/hr.

- High-dose rate (HDR) brachytherapy emits radiation at greater than $12 \mathrm{~Gy} / \mathrm{hr}$.

- Pulse-dose rate (PDR) brachytherapy involves short pulses of high-dose radiation, typically once per hour, to enhance the effectiveness of LDR.

Higher dose rates confer to shorter treatment times, and vice versa. With HDR brachytherapy, the implants are placed for only a few minutes at a time and then removed, with additional treatments given every several days or weeks. With LDR brachytherapy, the radioactive source may be left in for one or several days. Of all the dose rate options, HDR brachytherapy is the one most commonly used for breast cancer. When used on its own, HDR brachytherapy can reduce the treatment time from six to seven weeks for EBRT to just five days.

LDR and MDR brachytherapy are typically used to "boost" EBRT following surgery, while ULDR brachytherapy is reserved for permanent implants, including PBSI. PDR brachytherapy is a more recent innovation that combines the efficacy of HDR technology with the improved tissue safety of LDR brachytherapy.

\section{Procedure}

Brachytherapy requires a treatment team, which may include a radiation oncologist, dosimetrist, radiation therapist, nurse, and occasionally a surgeon. With the exception of NIBB, breast brachytherapy will involve some sort of invasive procedure. The radioactive source may be delivered using a needle-like applicator, via one or more temporary catheters, or during breast surgery. The implantation is usually performed in a hospital operating room specially designed to keep radiation from seeping out. Depending on the procedure, you will either be given local anesthesia to numb the general area or general anesthesia to put you to sleep. As with any procedure involving anesthesia, you will be given specific instructions as to when you may need to stop eating or drinking prior to the procedure.

\section{Throughout the Procedure:}

Breast brachytherapy requires an imaging tool, such as an X-ray, ultrasound, or computed tomography (CT), to direct the placement of the radioactive source. Once the coordinates are determined, one or more sources are strategically placed to target the cancer but spare the surrounding cells.

The various procedures can be broadly described as follows:

With interstitial brachytherapy, several catheters are placed so that radioactive pellets can be readily inserted and removed. This is most often done one to two weeks after surgery. HDR brachytherapy is delivered in a series of 10- to 20-minute sessions. LDR brachytherapy is delivered continuously over one to two days.

With intracavity brachytherapy, a single catheter with an inflatable balloon is inserted into the breast cavity following a lumpectomy. Tiny radioactive pellets are then fed into the balloon. Sometimes the catheter is placed during surgery and filled with pellets in the oncologist's office several days later.

With intraoperative brachytherapy, the radiation is delivered into the breast cavity via a targeted emitter immediately following the lumpectomy. The treatment is usually completed in less than an hour.

With PBSI, the radioactive seeds are individually placed with a percutaneous applicator that inserts the seeds directly through the skin.

\section{Following the Procedure:}

Following the completion of the brachytherapy procedure, you are led to the recovery room and monitored from anywhere from 30 minutes to several hours. You will likely feel localized pain around the implantation site, as well as swelling, redness, and bruising. Side effects from the anesthesia may include sleepiness, confusion, and nausea. Some radioactive implants may be left in place from one to several days. If so, you will probably need to remain in the hospital during treatment, often in a special radiationproof room. Larger implants may require you to stay in bed without moving. If you have been given permanent brachytherapy seeds, you can usually go home the same day. The radiation will wear off in a couple of weeks, and the seeds will gradually deteriorate over time. 


\section{Contraindications:}

Breast brachytherapy is not for everyone. Although most women with breast cancer will benefit from lumpectomy and EBRT, only a subgroup are appropriate candidates for breast brachytherapy. Some of the contraindications include Pregnancy, Stage 3 or stage 4 breast cancer, having bilateral breast cancer (cancer in both breasts), having had cancer in the opposite breast, having had other cancers (with the exception of skin cancer and certain gynecological cancers), Paget's disease (which disrupts the replacement of bone tissue) and certain connective tissue or collagen disorders. [119]

\subsubsection{Skin Cancer:}

The widespread availability of HDR remote afterloading brachytherapy units allows the use of surface molds as an alternative to electron beam and, for cases where surface irregularity, proximity to bone, or poor intrinsic tolerance of tissues do not allow for satisfactory treatment using electron beam. For most cases, a satisfactory mold can be made from 5-mm-thick sheets of wax, with the HDR catheters spaced 1$\mathrm{cm}$ apart. A simpler alternative is to use commercially available surface template applicators (e.g., Freiburg flab from Nucletron Corporation, Columbia, MD and HAM applicator from Mick Radionuclear Instruments Inc, Bronx, NY), which are used for intraoperative HDR brachytherapy. ${ }^{[120], ~[121] ~ T h e r e ~ i s ~ a ~ w i d e ~ r a n g e ~ o f ~}$ recommended doses and fractionation schemes for treating skin cancer. Doses in the range of 3500 cGy in five fractions to $5000 \mathrm{cGy}$ in ten fractions have been used with success in HDR molds. Standard, more prolonged fractionation schemes with 180-200 cGy daily or twice daily fractions can also be used. The linear quadratic radiobiological model can be used to determine the total dose for a given fractionation scheme. [100]

\subsubsection{Lung Cancer:}

Lung cancer remains a major health problem worldwide. Although the disease had a lower incidence over a hundred years ago, the widespread dissemination and availability of tobacco products has led to a rapid increase in the worldwide incidence and mortality of lung cancer. The best reported five year survival rates are only $15 \%$ in developed countries. ${ }^{[122]}$

For patients with stage III non-small-cell lung cancer and a reasonable performance status, the treatment of choice is concurrent chemoradiotherapy. ${ }^{[123]}$ Approximately 40-50\% of patients with lung cancer experience malignant airway occlusion. Retrospective data show a high response rate when HDR BT is used for palliation. Clinical end points are time to reaeration, success of reaeration and relief of post-obstructive symptoms, such as chest pain, shortness of breath, cough and haemoptysis. [124] The risk of fatal haemoptysis is approximately $10 \%$, with some series reporting higher rates. $[125,126]$ Risk factors include upper lobe lesions, which are in close proximity to the pulmonary artery, repeated endoscopies when HDR BT is performed weekly and retreatment after definitive EBRT. In addition, the irradiated volume has been identified as a major risk factor that concerns life threatening haemorrhage after endobronchial HDR BT. ${ }^{[127]}$ The application is usually performed jointly with a pulmonologist and requires endobronchial placement of one or more small sized catheters (diameter of 5 or 6 French), using a flexible fibre optic bronchoscope. These catheters can be left in place for either a few hours or a couple of days, depending on the selection of fractionation schedules. The University of Wisconsin, United States of America, reported an approximate $80 \%$ palliating rate of obstructive symptoms when using a single placement followed by fractionated HDR BT over two days, with the catheter left in place. Four fractions of $4 \mathrm{~Gy}$ each were delivered twice daily to yield an LDR equivalent of $20 \mathrm{~Gy}$. ${ }^{[128]}$ Another feasible fractionated HDR BT schedule recommends a total dose of $18 \mathrm{~Gy}$ given in three fractions (6 Gy each) over two days, which results in a shorter duration of the catheter, and $20 \mathrm{~Gy}$ in four fractions of $5 \mathrm{~Gy}$ each at one per week. ${ }^{[124]}$ The dose is prescribed at $10 \mathrm{~mm}$ from the source axis when small applicators are used or at $5 \mathrm{~mm}$ from the applicator surface for large applicators. [92] Some authors have retrospectively compared their results regarding the use of HDR versus LDR BT for endobronchial obstruction palliation. The results showed similar response rates in terms of symptom control and adverse effect incidences. Hence, they recommend that the HDR technique should progressively replace the LDR technique because the first offers advantages such as eliminating a hospital stay, improved convenience for the patient and radiation safety for personnel. ${ }^{[125]}$ HDR BT is an effective palliative treatment for selected patients who suffer from symptomatic endobronchial central relapse after EBRT. ${ }^{[129]}$ For these cases, 60-70\% response rates have been reported, although the incidence of fatal adverse effects could be high. [125] In conclusion, HDR endobronchial BT effectively palliates malignant airway occlusion and should be considered, when feasible. 


\subsection{Clinical advances in high dose rate brachytherapy:}

Current indications for HDR BT have been shown that some of the recent clinical advances in this field should be considered when planning a new HDR program. One way to improve the therapeutic ratio of HDR BT is to deliver irradiation during surgery while the patient is still under anaesthesia. This technique (intraoperative HDR BT) allows radiosensitive normal tissue to be retracted or shielded during surgery, which lowers the radiation dose to normal tissue. ${ }^{[130]}$ In addition, because irradiation is given under direct vision, the risk of a geographical miss is reduced. Best achievable tumor debulking is an aim. The tumor bed is irradiated using special intraoperative applicators with parallel HDR catheters embedded in them, at least $1 \mathrm{~cm}$ apart. The use of a fixed geometry applicator allows the patient to be treated without delay, using a preplanned atlas or library for the selected applicator. Doses of 10-20 Gy are usually given as a single fraction over $10-60 \mathrm{~min}$. Ideally, the surgery should be performed in a shielded operating room with remote anaesthesia and a television monitoring system. Publications on intraoperative HDR BT in rectal cancer, soft tissue sarcomas, and head and neck cancer are available. ${ }^{[131]}$ When starting a new programme, a shielded operating room with appropriate imaging facilities (e.g. mobile C-arm or mobile cone beam CT unit) should be integrated into the plan, if intraoperative HDR BT is considered. Because of the restricted availability of fully equipped and shielded operating rooms, only a limited number of institutions perform intraoperative HDR BT. [130] Some HDR devices are certified as transportable radioactive containers. This approach allows different hospitals to use an HDR afterloader on a shared basis in cases in which centres do not have a sufficient patient load to justify the purchase of individual devices. The development of miniature sources allows for percutaneous interstitial BT through very thin needles (21gauge). This approach could be of particular advantage for the treatment of lip, nose and eyelid tumors and for percutaneous image guided treatment of intrathoracic or intra-abdominal tumors.

\section{DISCUSSION}

Brachytherapy has slowly evolved over the past century. At first, brachytherapy did not gain much popularity, because it was performed by inserting the radioactive sources directly into the tumor ("hot" loading), which exposed personnel to high doses of radiation. Manual afterloaded procedures significantly reduced radiation exposure to personnel, in which hollow catheters are initially inserted into the tumor and then loaded with radioactive sources after proper positioning. In remote afterloading, an operator loaded the radioactive material into the catheters using remote control outside the room. In highdose-rate brachytherapy, outpatient brachytherapy is allowed by the elimination of the radiation exposure and the short treatment times. A single dose of radiation to be delivered during surgery is allowed by intraoperative HDR brachytherapy. Doses of 10-20 Gy are usually given as a single fraction over $10-60 \mathrm{~min}$. The advantages of intraoperative HDR brachytherapy over electron beam IORT or perioperative brachytherapy are listed in Table 3.8 . Unfortunately, the relative scarcity of shielded operating rooms has currently limited its availability to just a few centers. ${ }^{[132,133,134]}$

Case selection and proper patient evaluation are essential, although brachytherapy is a very effective modality. If the tumor is widely metastatic or very large, one is doomed to fail due to the biology of the tumor in the latter case and due to the physics of dose distribution in the former case. The various brachytherapy modalities have some differences between them and are mentioned in (Table 3.8). When selecting the brachytherapy modality in a particular situation, then these differences should be kept in mind.

Table 3.8. Comparison of different brachytherapy techniques, LDR low-dose-rate brachytherapy, MDR medium-dose-rate brachytherapy, PDR pulsed dose rate, HDR high-dose-rate brachytherapy, IOHDR intraoperative high dose rate, EBRT external beam radiation therapy ${ }^{[16]}$

\begin{tabular}{|l|l|l|l|l|l|l|}
\hline & \multicolumn{2}{l}{ LDR Ir-192 LDR remote } & MDR & PDR & HDR & IOHDR \\
\hline Dose rate & low & low & medium & high & high & high \\
\hline Duration of each treatment & $2-6$ days & $2-4$ days & 1 days & minutes & minutes & minutes \\
\hline Overall duration of treatment & $2-6$ days & $2-4$ days & 1 days & $2-4$ days & $3-5$ weeks & minutes \\
\hline Radiation hazards & high & small & small & small & small & small \\
\hline Availability (worldwide) & ++ & - & - & - & + & - \\
\hline Ease of optimization & - & - & - & + & + & + \\
\hline Dose as sole modality (Gy) & 60 & 60 & 40 & 60 & $30-40$ & $15-20$ \\
\hline
\end{tabular}


High-dose-rate has special relevance for developing countries, where resources may be rare. In this regard, in developing countries, the International Atomic Energy Agency has recently issued recommendations for the use of HDR brachytherapy. ${ }^{[135]}$

A brief summary is given here; A HDR treatment system should be purchased as a complete unit that includes the source loading unit, control console, treatment planning system, applicators and 192Ir radioactive source. Infrastructure support may require improved buildings or additional and access to or procurement of new imaging facilities. For the annual maintenance necessary to keep the system operational and quarterly source replacement, a supportive budget is needed. Before HDR can be introduced the radiation oncologist, medical physicist, and technologist should be specially trained as new techniques or sites of treatment are introduced, therefore the training for the oncologist and medical physicist is an ongoing process. Procedures must be introduced for the planning system and quality assurance of patient treatment. Emergency procedures with adequate training of all associated personnel must be in place. The decision to select HDR in preference to alternate methods of brachytherapy is influenced by the ability of the machine to treat a wide variety of clinical sites. In departments with personnel and budgetary resources to support this equipment appropriately, economic advantage becomes evident only if large numbers of patients are treated. With HDR, it is possible to treat a large number of patients in institutions that have a high volume of brachytherapy patients but insufficient in-patient facilities for LDR brachytherapy or insufficient finances for the purchase of iodine-125 or palladium seeds for permanent implants. Intangible benefits of source safety, personnel safety, and easy adaptation to fluctuating demand for treatments also require consideration when evaluating the need to introduce this treatment system.

When HDR brachytherapy is used, the treatments must be executed carefully, because the short treatment times do not allow any time for correction of errors, and mistakes can result in harm to patients. Hence, it is very important that all personnel involved in HDR brachytherapy be well trained and be constantly alert. However, with proper case selection and delivery technique, HDR brachytherapy has great promise and convenience, because it eliminates radiation exposure, allows short treatment times, and can be performed on an outpatient basis.

It is expected that the use of HDR brachytherapy will greatly expand over the next decade and that refinements will occur primarily in the integration of imaging (computed tomography, magnetic resonance imaging, intraoperative ultrasonography) and optimization of dose distribution. ${ }^{[136]}$ It is anticipated that better tumor localization and normal tissue definition will help to optimize dose distribution to the tumor and reduce normal tissue exposure. [137] The development of well-controlled randomized trials addressing issues of efficacy, toxicity, quality of life, and costs versus benefits will ultimately define the role of HDR brachytherapy in the therapeutic armamentarium.

\section{CONCLUSION}

HDR brachytherapy is widely used to treat head and neck cancer, prostate cancer, lung cancer, esophagus cancer, skin cancer, breast cancer, cervix cancer and carcinoma of the endometrium. HDR brachytherapy is an important treatment for carcinoma prostate. HDR brachytherapy can be used for monotherapy for low risk patients and boost for intermediate/high risk patients. It provides excellent local control. It preserves sexual function. High dose rate brachytherapy produced a more conformal plan and provides higher mean dose to prostate. Brachytherapy is effective in achieving dose escalation. Taking only a few minutes, the use of HDR has the added advantage of the treatments allowing them to be given on an outpatient basis with minimal risk of applicator movement and minimal patient discomfort. $90 \%$ of oesophageal cancers are locally advanced at diagnosis, and treatment yields discouraging results. High dose rate brachytherapy (HDR-BT) permits an increment of local doses without a significant increment of toxicity. When HDR brachytherapy is used, the treatments must be executed carefully, because the short treatment times do not allow any time for correction of errors, and mistakes can result in harm to patients. However, with proper case selection and delivery technique, HDR brachytherapy has great promise and convenience, because it eliminates radiation exposure, allows short treatment times, and can be performed on an outpatient basis. HDR brachytherapy can deliver a big dose with limiting the exposure of the surrounding normal tissues and is the utmost precision radiation therapy. Without real observation and palpation of the tumor and the patient, HDR brachytherapy cannot be performed. HDR brachytherapy is the most precision radiation therapy even more better than carbon ion therapy. At the time of invasive placement of the radiation source into the tumor area, brachytherapy requires skills and techniques of radiation oncologists. Demand for oncology services is expected to rise rapidly, driven by the aging and growth of the population and improvements in cancer survival rates, at the 
same time the oncology workforce is aging and retiring in increasing numbers. Demand is expected to rise $48 \%$ between 2005 and 2020 . The supply of services provided by oncologists during this time is expected to grow more slowly, approximately $14 \%$, based on the current age distribution and practice patterns of oncologists and the number of oncology fellowship positions. This translates into a shortage of 9.4 to 15.0 million visits, or 2,550 to 4,080 oncologistsroughly one-quarter to one-third of the 2005 supply. The baseline projections do not include any alterations based on changes in practice patterns, service use, or cancer treatments. Various alternate scenarios were also developed to show how supply and demand might change under different assumptions. Among the options to consider are increasing the number of oncology fellowship positions, increasing use of non-physician clinicians, increasing the role of primary care physicians in the care of patients in remission, and redesigning service delivery.

\section{REFERENCE}

1. Nag S, Samsami N Pitfalls of inappropriate optimization. J Brachyther Int 2000;16: 187-198.

2. International Atomic Energy Agency, Setting Up a Radiotherapy Programme: Clinical, Medical Physics, Radiation Protection and Safety Aspects, IAEA, Vienna 2008.

3. Annede P, Dumas I, Schernberg A, Tailleur A, Fumagalli I, Bockel $\mathrm{S}$, et al. Radiobiological optimization comparison between pulse-dose-rate and high-dose-rate brachytherapy in patients with locally advanced cervical cancer. Brachytherapy 2019;18(3):370-7.

4. International commission on radiation units and measurements, Dose and Volume Specification for Reporting Intracavitary Therapy in Gynecology, ICRU Rep. 38, ICRU, Bethesda, MD, 1985.

5. American Cancer Society. Getting Internal Radiation Therapy (Brachytherapy). Accessed 7/31/2020.

6. Arthur DW, Vicini FA, Kuske RR, Wazer DE, Nag S. Accelerated partial breast irradiation: an updated report from the American Brachytherapy Society. Brachytherapy 2003;2(2):124-30.

7. Nag S, Erickson B, Parikh S, Gupta N, Varia M, Glasgow G. The American Brachytherapy Society recommendations for high-dose-rate brachytherapy for carcinoma of the endometrium. International Journal of Radiation Oncology Biology Physics 2000;48(3):779-90.
8. Patel RR, Arthur DW. Hematology/ Oncology Clinics of North America 2006;20(1):97-118.

9. National Cancer Institute. Available at: www.cancer.gov/cancertopics/factsheet/ Therapy/radiation; Accessed 2 February 2011.

10. Stewart AJ,Jones B. In: Brachytherapy: applications and techniques. Devlin PM (Ed). Philadelphia, PA, LWW. 2007.

10a. National Cancer Institute. Available at: www.cancer.gov/cancertopics/factsheet/ Therapy/radiation. Accessed 2 February 2011.

10b. Viswanathan AN, Petereit DG. In: Brachytherapy: applications and techniques. Devlin PM (Ed). Philadelphia, PA, LWW. 2007.

10c. Potters L, Morgenstern C, Calugaru E, Fearn P, Jassal A, Presser J, Mullen E. 12-year outcomes following permanent prostate brachytherapy in patients with clinically localized prostate cancer, The Journal of urology 2005;173(5):1562-1566.

11. Lawrence TS, Ten Haken RK, Giaccia A. In: Cancer: Principles and Practice of Oncology. 8th ed.De Vita VT Jr., Lawrence TS, Rosenberg SA (Eds). Phil-adelphia, PA, LWW. 2008.

12. Mac Millan Cancer Support. Available at: www.macmillan.org.uk/Cancerinformati on/Cancertreatment/Treatmenttypes/Surger y/Generalinformation/Whatitisusedfor. Asp x.Accessed 2 February 2011.

13. National Cancer Institute. Available at: www.cancer.gov/cancertopics/chemothe rapy-and-you/; Accessed 2 February 2011.

14. Fu K, Phillips T. High-dose-rate versus low-dose-rate intracavitary brachytherapy for carcinoma of the cervix. Int $\mathbf{J}$ Radiat Oncol Biol Phys 1990;19:791-796.

15. Orton CG, Seyedsadr M, Somnay A. Comparison of high and low dose rate remote afterloading for cervix cancer and the importance of fractionation. Int $\mathrm{J}$ Radiat Oncol Biol Phys 1991;21:1425-1434.

16. Nag S, Scruggs, GR. Clinical applications of high-dose-rate brachytherapy. In Technical Basis of Radiation Therapy Springer, Berlin, Heidelberg.2011; 461-484.

17. Nag S, Erickson B, Thomadsen B, Orton C, Demanes JD, Petereit D. The American Brachytherapy Society recommendations for HDR brachytherapy of the cervix. Int $\mathbf{J}$ Radiat Oncol Biol Phys 2000b; 48:201-211.

18. Nakano T, Kato S, Ohno T, Tsujii H, Sato S, Fukuhisa K, Arai, T. Long-term results of high-dose rate intracavitary brachytherapy for squamous cell carcinoma of the 
uterine cervix. Cancer, 2004; 103(1), 92101.

19. Patel FD, Rai B, Mallick I, Sharma SC High dose rate brachytherapy in uterine cervical carcinoma. Int J Radiat Oncol Biol Phys 2005; 62:125-130.

20. Shigematsu Y, Nishiyama K, Masaki N, Inoue $\mathrm{T}$. Treatment of carcinoma of the uterine cervix by remotely controlled afterloading intracavitary radiotherapy with highdoes rate: a comparative study with a low-dose rate system. Int J Radiat Oncol Biol Phys 1983; 9:351-356.

21. Mandell LM, Nori D, Anderson LL, Hilaris BS. Postoperative vaginal radiation in endometrial cancer using a remote afterloading technique. Int $\mathbf{J}$ Radiat Oncol Biol Phys 1985;11:473-478.

22. Lybeert MLM, van Putten WLJ, Ribot JG, Crommelin MA. Endometrial carcinoma: high dose rate brachytherapy in combination with external irradiation a multivariate analysis of relapses. Radiother Oncol 1989; 16:245-252.

23. Sorbe BG, Smeds AC. Postoperative vaginal irradiation with high dose rate afterloading technique in endometrial carcinoma stage I. Int J Radiat Oncol Biol Phys 1990;18:305-314.

24. Sorbe B, Frankendal B. Intracavitary irradiation of endometrial carcinoma stage I by a high dose rate afterloading technique. Gynecol Oncol 1989; 33:135-145.

25. Rouanet P, Dubois J, Gely S, Pourquier H. Exclusive radiation therapy in endometrial carcinoma. Int J Radiat Oncol Biol Phys 1993;26(2), 223-228.

26. Nag S. Modern techniques of radiation therapy for endometrial cancer. Clin highdose rate brachytherapy as monotherapy for the treatment of favorable stage prostate cancer: a feasibility report. Int J Radiat Oncol Biol Phys 1996; 49:61-69.

27. Mehta M, Petereit D, Chosy L, Harmon M, Fowler J, Shahabi S, Thomadsen B, Kinsella T. Sequential comparison of low dose rate and hyperfractionated high dose rate endobronchial radiation for malignant airway occlusion. Int J Radiat Oncol Biol Phys 1992;23(1):133-9.

28. Mehta MP, Lamond JP, Nori D, Speiser BL Brachytherapy of lung cancer. In: Nag S (ed). Principles and practice of brachytherapy. Futura Publishing Co., Armonk, NY, 1997; 323-349.

29. Speiser BL. Brachytherapy in the treatment of thoracic tumors. Lung and esophageal. Hematol Oncol Clin North Am 1999;13: 609-634.
30. Gaspar LE. Brachytherapy in lung cancer. J Surg Oncol 1998;67:60-70.

31. Nag S, Kelly JF, Horton JL, Komaki R, Nori D. The American Brachytherapy Society recommendations for HDR brachytherapy for carcinoma of the lung. Oncology 2001a;15:371-381.

32. Speiser B, Spratling L. Fatal hemoptysis: complication or failure of treatment. Int $\mathbf{J}$ Radiat Oncol Biol Phys 1993a;25:925.

33. Speiser BL, Spartling L. Radiation bronchitis and stenosis secondary to high dose rate endobronchial irradiation. Int $\mathbf{J}$ Radiat Oncol Biol Phys 1993b;25:589-597.

34. Khanavkar B, Stern P, Alberti W, Nakhosteen JA. Complications associated with brachytherapy alone or with laser in lung cancer. Chest 1991; 99:1062-1065.

35. Bedwinek J, Petty A, Bruton C, Sofield, J, Lee L. The use of high dose rate endobronchial brachytherapy to palliate symptomatic endobronchial recurrence of previously irradiated bronchogenic carcinoma. Int J Radiat Oncol Biol Phys 1992;22 (1), 23-30.

36. Aygun $\mathrm{C}$, Weiner $\mathrm{S}$, Scariato $\mathrm{A}$ et al. Treatment of nonsmall cell lung cancer with external beam radiotherapy and high dose rate brachytherapy. Int J Radiat Oncol Biol Phys 1992;23:127-132.

37. Huber RM, Fischer R, Haútmann $\mathrm{H}$ et al. Does additional brachytherapy improve the effect of external irradiation? A prospective, randomized study in central lung tumors. Int J Radiat Oncol Biol Phys 1997;38:533540.

38. Perol M, Caliandro R, Pommier P, Malet C. Curative irradiation of limited endobronchial carcinomas with highdoserate brachytherapy. Results of a pilot study. Chest 1997;111:1417-1423.

39. Furuta M, Tsukiyama I, Ohno $\mathrm{T}$ et al. Radiation therapy for roentogenographically occult lung cancer by external beam irradiation and endobronchial high dose rate brachytherapy. Lung Cancer 1999;25:183189.

40. Saito M, Yokoyama A, Kurita Y, Uematsu T, Tsukada H, Yamanoi T. Treatment of roentgenographically occult endobronchial with external beam radiotherapy and intraluminal low dose rate brachytherapy: second report. Int J Radiat Oncol Biol Phys 2000;47:673-680.

41. Marsiglia H, Baldeyrou P, Lartigau E et al. High-doserate brachytherapy as a sole modality for early-stage endobronchial carcinoma. Int $\mathbf{J}$ Radiat Oncol Biol Phys 2000;47:665-672. 
42. Macha HN, Wahlers B, Reichle C, von Zwehl. Endobronchial radiation therapy for obstructing malignancies: ten years' experience with iridium-192 high-dose radiation brachytherapy afterloading technique in 365 patients. Lung 1995; 173:271-280

43. Hishikawa Y, Kamikonya N, Tanaka S, Miura T. Radiotherapy of esophageal carcinoma: role of high dose rate intracavitary irradiation. Radiother Oncol 1987; 9:13-20.

44. Sur RK, Levin CV, Donde B et al. Prospective randomized trial of HDR brachytherapy as a sole modality in palliation of advanced esophageal carcinoma an international atomic energy agency study. Int J Radiat Oncol Biol Phys 2002; 53:127-133.

45. Sur R, Donde B, Falkson $\mathrm{C}$ et al. Randomized prospective study comparing high dose rate intraluminal brachytherapy (HDRILBT) alone to HDRILBT and external beam radiotherapy in the palliation of advanced esophageal cancer. Brachytherapy 2004;3:191-195.

46. Smith EC, others Nature Reviews Disease Primers Volume 3: 2018; 17048.

47. Dinges S, Deger S, Koswig S, Boehmer D. High-dose rate interstitial with external beam irradiation for localized prostate cancer-results of a prospective trial. Radiother Oncol 1998;48:197-202.

48. Martinez A, Kestin L, Stromberg J. Interim report of image guided conformal high dose rate brachytherapy for patients with unfavorable prostate cancer: the William Beaumont phase II dose escalating trial. Int J Radiat Oncol Biol Phys 2000; 47:343352.

49. Martinez A, Pataki I, Edmundson G, et al. Phase II prospective study of the use of conformal dose rate endobronchial radiation for malignant airway occlusion. Int J Radiat Oncol Biol Phys 2001; 23:133-139.

50. Galalae RM, Martinez A, Mate T, et al. Long-term outcome by risk factors using conformal high dose rate brachytherapy (HDR-BT) boost with or without neoadjuvant androgen suppression for localized prostate cancer. Int J Radiat Oncol Biol Phys 2004;58:1048-1055.

51. Astrom L, Pederson D, Mercke C, et al. Long-term outcome of high dose rate brachytherapy in radiotherapy of localised prostate cancer. Radiother Oncol 2005;74: 157-161.

52. Edmundson GK, Yan D, Martinez A. Intraoperative optimization of needle placement and dwell times for conformal prostate brachytherapy. Int J Radiat Oncol Biol Phys 1995;33:1257-1264.

53. Duchesne GM, Peters LJ. What is the $\alpha / \beta$ ratio for prostate cancer? Rationale for hypofractionated high-dose-rate brachytherapy. Int J Radiat Oncol Biol Phys 1999;44: 747-748.

54. Fowler J, Chappell R, Ritter M. Is $\alpha / \beta$ for prostate tumors really low? Int J Radiat Oncol Biol Phys 2001;50:1021-1031.

55. Chen RC, Basak R, Meyer AM, et al. Association between choice of radical prostatectomy, external beam radiotherapy, brachytherapy, or active surveillance and patient-reported quality of life among men with localized prostate cancer. JAMA 2017; 317:1141-1150.

56. Demanes JD, Rodriguez RR, Schour L, et al. High dose rate intensity-modulated brachytherapy with external beam radiotherapy for prostate cancer: California Endocurietherapy's 10 year results. Int J Radiat Oncol Biol Phys 2005;61:13061316.

57. Grills IS, Martinez AA, Hollander M, et al. High-dose rate brachytherapy as prostate cancer monotherapy reduces toxicity compared to low dose rate palladium seeds. J Urology 2004;171:1098-1104.

58. Yoshioka Y, Nose $\mathrm{T}$, Yoshida $\mathrm{K}$, et al. High-dose rate brachytherapy as monotherapy for localized prostate cancer: a retrospective analysis with special focus on tolerance and chronic toxicity. Int J Radiat Oncol Biol Phys 2003;56:213-220.

59. Kibel A. Screening and prostate-cancer mortality in a randomized European study. Yearbook of Medicine 2009; 173-174.

60. Kibel, A. Mortality results from a randomized prostate-cancer screening trial. Yearbook of Urology, 2009, 145-147.

61. United States Preventive Services Task Force, Screening for prostate cancer: US Preventive Services Task Force recommenddation statement, Ann. Intern. Med. 2008;149:185-191.

62. Smalley SR. The effect of dose on local control of prostate cancer. Int $\mathrm{J}$ Radiat Oncol Biol Phys. 1989;17(3):697-8.

63. Zelefsky MJ, Leibel SA, Gaudin PB, Kutcher GJ, Fleshner NE, Venkatramen ES, Reuter VE, Fair WR, Ling CC, Fuks Z. Dose escalation with three-dimensional conformal radiation therapy affects the outcome in prostate cancer. Int $\mathrm{J}$ Radiat Oncol Biol Phys 1998;41(3):491-500.

64. National Comprehensive Cancer Network, NCCN Guidelines for Treatment of Prostate Cancer, NCCN, Fort Washington, PA 2013. 
65. Ali AS, Hamdy FC, The spectrum of prostate cancer care: From curative intent to palliation, Curr Urol Rep 2007; 8;245-252.

66. Kovács G, Pötter R, Loch T, Hammer J, Kolkman-Deurloo IK, de la Rosette JJ, Bertermann H. GEC/ESTRO-EAU recommendations on temporary brachytherapy using stepping sources for localised prostate cancer. Radiother Oncol 2005;74 (2):137-48.

67. Aström L, Pedersen D, Mercke C, Holmäng $\mathrm{S}$, Johansson KA. Long-term outcome of high dose rate brachytherapy in radiotherapy of localised prostate cancer. Radiother Oncol 2005;74(2):157-61.

68. Martinez A, Gonzalez J, Spencer W, Gustafson G, Kestin L, Kearney D, Vicini FA. Conformal high dose rate brachytherapy improves biochemical control and cause specific survival in patients with prostate cancer and poor prognostic factors. J Urol 2003;169(3):974-80.

69. Pellizzon AC, Salvajoli JV, Maia MA, Ferrigno R, Novaes PE, Fogarolli RC, Pellizzon RJ. Late urinary morbidity with high dose prostate brachytherapy as a boost to conventional external beam radiation therapy for local and locally advanced prostate cancer. J Urol 2004;171(3):1105-8.

70. Sullivan L, Williams SG, Tai KH, Foroudi F, Cleeve L, Duchesne GM. Urethral stricture following high dose rate brachytherapy for prostate cancer. Radiother Oncol. 2009;91(2):232-6.

71. Ebara S, Manabe D, Kobayashi Y, Tanimoto R, Saika T, Nasu Y, Saito S, Satoh T, Miki K, Hashine K, Kumon H. The efficacy of neoadjuvant androgen deprivation therapy as a prostate volume reduction before brachytherapy for clinically localized prostate cancer. Acta Med Okayama. 2007;61(6):335-40.

72. Yoshioka Y, Nose T, Yoshida K, Inoue T, Yamazaki H, Tanaka E, Shiomi H, Imai A, Nakamura S, Shimamoto S, Inoue T. Highdose-rate interstitial brachytherapy as a monotherapy for localized prostate cancer: treatment description and preliminary results of a phase I/II clinical trial. Int J Radiat Oncol Biol Phys. 2000;48(3):675-81.

73. Martinez AA, Pataki I, Edmundson G, Sebastian E, Brabbins D, Gustafson G. Phase II prospective study of the use of conformal high-dose-rate brachytherapy as monotherapy for the treatment of favorable stage prostate cancer: a feasibility report. Int J Radiat Oncol Biol Phys. 2001;49(1): 61-9.

74. Martin T, Baltas D, Kurek R, Röddiger S, Kontova M, Anagnostopoulos G, Dannen- berg T, Buhleier T, Skazikis G, Tunn U, Zamboglou N. 3-D conformal HDR brachytherapy as monotherapy for localized prostate cancer. A pilot study. Strahlenther Onkol. 2004;180(4):225-32.

75. Dörr W, Jaal J, Zips D. Prostate cancer: Biological dose considerations and constraints in tele- and brachytherapy, Strahlenther. Onkol 2007;183:14-15.

76. Corner C, Rojas AM, Bryant L, Ostler P, Hoskin P. A Phase II study of high-doserate afterloading brachytherapy as monotherapy for the treatment of localized prostate cancer. Int $\mathrm{J}$ Radiat Oncol Biol Phys. 2008;72(2):441-6.

77. Yoshioka Y, Konishi K, Sumida I, Takahashi Y, Isohashi F, Ogata T, Koizumi M, Yamazaki H, Nonomura N, Okuyama A, Inoue T. Monotherapeutic high-dose-rate brachytherapy for prostate cancer: five-year results of an extreme hypofractionation regimen with $54 \mathrm{~Gy}$ in nine fractions. Int $\mathbf{J}$ Radiat Oncol Biol Phys. 2011;80(2):469-75.

78. Martinez AA, Demanes J, Vargas C, Schour L, Ghilezan M, Gustafson GS. High-doserate prostate brachytherapy: an excellent accelerated-hypofractionated treatment for favorable prostate cancer. Am J Clin Oncol. 2010;33(5):481-8.

79. Demanes DJ, Martinez AA, Ghilezan M, Hill DR, Schour L, Brandt D, Gustafson G. High-dose-rate monotherapy: safe and effective brachytherapy for patients with localized prostate cancer. Int J Radiat Oncol Biol Phys. 2011;81(5):1286-92.

80. Konishi K, Yoshioka Y, Isohashi F, Sumida I, Kawaguchi Y, Kotsuma T, Adachi K, Morimoto M, Fukuda S, Inoue $\mathrm{T}$. Correlation between dosimetric parameters and late rectal and urinary toxicities in patients treated with high-dose-rate brachytherapy used as monotherapy for prostate cancer. Int $\mathrm{J}$ Radiat Oncol Biol Phys. 2009;75(4):1003-7.

81. Yoshioka Y, Nose $\mathrm{T}$, Yoshida $\mathrm{K}$, et al. High-dose rate brachytherapy as monotherapy for localized prostate cancer: a retrospective analysis with special focus on tolerance and chronic toxicity. Int $\mathbf{J}$ Radiat Oncol Biol Phys 2003;56:213-220.

82. Simnor T, Li S, Lowe G, Ostler P, Bryant L, Chapman C, Inchley D, Hoskin PJ. Justification for inter-fraction correction of catheter movement in fractionated high dose-rate brachytherapy treatment of prostate cancer. Radiother Oncol 2009;93(2):253-8.

83. Lee $B$, Shinohara $K$, Weinberg V, Gottschalk AR, Pouliot J, Roach M 3rd, Hsu IC. Feasibility of high-dose-rate 
brachytherapy salvage for local prostate cancer recurrence after radiotherapy: the University of California-San Francisco experience. Int J Radiat Oncol Biol Phys 2007;67(4):1106-12.

84. Tharp M, Hardacre M, Bennett R, Jones WT, Stuhldreher D, Vaught J. Prostate high-dose-rate brachytherapy as salvage treatment of local failure after previous external or permanent seed irradiation for prostate cancer. Brachytherapy. 2008;7(3): 231-6.

85. Slessinger ED. Practical considerations for prostate HDR brachytherapy, Brachytherapy 2010;9: 282-287.

86. Cury FL, Duclos M, Aprikian A, Patrocinio H, Souhami L. Prostate gland edema after single-fraction high-dose rate brachytherapy before external beam radiation therapy. Brachytherapy. 2010;9(3):208-12.

87. Mueller A, Wallner K, Corriveau J, Arthurs S, Gwinn M, Sutlief S. A reappraisal of local anesthesia for prostate brachytherapy. Radiother Oncol. 2003;67(3):309-12.

88. Wallner K. Prostate brachytherapy under local anesthesia: Lessons from the first 600 patients, Brachytherapy 2002; 1:145-148.

89. Pellizzon AC, Salvajoli JV, Novaes PE, Maia MA, Ferigno R, Fogaroli RC. Needle displacement during high-dose-rate afterloading brachytherapy boost and conventional external beam radiation therapy for initial and local advanced prostate cancer. Urol Int. 2003;70(3):200-4.

90. Kovács G, Melchert C, Sommerauer M, Walden O. Intensity modulated high-doserate brachytherapy boost complementary to external beam radiation for intermediateand high-risk localized prostate cancer patients--how we do it in Lübeck/Germany. Brachytherapy. 2007;6(2):142-8.

91. Donath D, Vuong T, Shnouda G, et al. The potential uses of high-dose-rate brachytherapy in patients with head and neck cancer. Eur Arch Otorhinolaryngol 1995; 252:321-354.

92. Inoue $\mathrm{T}$, Inoue $\mathrm{T}, \mathrm{Teshima} \mathrm{T}$, Murayama $\mathrm{S}$. Phase III trial of high and low dose rate interstitial radiotherapy for early oral tongue cancer. Int J Radiat Oncol Biol Phys 1996;36:1201-1204.

93. Lau HY, Hay JH, Flores AD, Threlfall WJ. Seven fractions of twice daily high doserate brachytherapy for node-negative carcinoma of the mobile tongue results in loss of therapeutic ratio. Radiother Oncol. $1996 ; 39(1): 15-8$.

94. Yu L, Vikram B, Chadha M. High dose rate interstitial brachytherapy in patients with cancers of the head and neck. Endocuriether Hyperther Oncol 1996;12:1-6.

95. Dixit S, Baboo HA, Rakesh V. Interstitial high dose rate brachytherapy in head and neck cancers: preliminary results. J Brachyther Int 1997;13:363-370.

96. Leung TW, Wong VYW, Wong CM, et al, Technical hints for high dose rate interstitial tongue brachytherapy. Clin Oncol 1998;10: 231-236.

97. Gao Li, Xu Guo-zhen, Yin Wei-b,o et al. Preliminary experience in HDR brachytherapy for 72 nasopharyngeal carcinoma patients. In: Mould RF (ed) Brachytherapy in the Peoples Republic of China. Kowloon, Nucletron Far East,1992; E76-E81.

98. Levendag PC, Vikram B, Flores AD, et al. High dose rate brachytherapy for cancer of the head and neck. In: Nag S (ed). High dose rate brachytherapy: a textbook. Futura Publishing Co., Armonk, NY, 1994; 23727.

99. Jolly DE, Nag S Technique for construction of dental molds for high-dose-rate remote brachytherapy. Spec Care Dentist 1992;12: 219-224.

100. Nag S, Vikram B, Demanes JD, Cano E, Puthawala AA. The American Brachytherapy Society Recommendations for HDR Brachytherapy for Head and Neck Carcinoma. Int. J. Radiat. Oncol. Biol. Phys 2001;50: 1190-1198.

101. Nag S, Gupta N. A simple method of obtaining equivalent doses for use in HDR brachytherapy. Int J Radiat Oncol Biol Phys 2000;46:507-513.

102. Szewczyk MP, Pazdrowski J, DańczakPazdrowska A, et al. Analysis of selected recurrence risk factors after treatment of head and neck basal cell carcinoma. Postep Derm Alergol 2014;31:146-51.

103. Nag S, Koc M, Schuller D, Tippin D, Grecula JC. Intraoperative single fraction high dose rate brachytherapy for head and neck cancers. Brachytherapy 2005;4:217223.

104. Harrison LB, Franzese F, Gaynor JJ, Brennan MF. Longterm results of a prospective randomized trial of adjuvant brachytherapy in the management of completely resected soft tissue sarcomas of the extremity and superficial trunk. Int $\mathbf{J}$ Radiat Oncol Biol Phys 1992;27:259-265.

105. Alektiar KM, Leung D, Zelefsky MJ et al (2002) Adjuvant brachytherapy for primary high-grade soft tissue sarcoma of the extremity. Ann Surg Oncol 9:48-56.

106. Alekhteyar KM, Porter AT, Herskovic AM. Preliminary results of hyperfractionated high dose rate brachytherapy in soft tissue 
sarcoma. Endocuriether Hypertherm Oncol 1994;10:179-184.

107. Crownover RL, Marks KE, Zehr RJ. Initial results with high dose rate brachytherapy for soft-tissue sarcomas. Sarcoma 1997;1: 196-205.

108. Koizumi M, Inoue $\mathrm{T}$, Yamazaki $\mathrm{H}$ et al. Perioperative fractionated high-dose rate brachytherapy for malignant bone and soft tissue tumors. Int J Radiat Oncol Biol Phys 1999;43:989-993.

109. Alektiar KM, Hu K, Anderson L, et al. High dose rate intraoperative radiation therapy (HDR-IORT) for retroperitoneal sarcomas. Int J Radiat Oncol Biol Phys 2000;47:157163.

110. Nag S, Shasha D, Janjan N, Petersen I, Zaider M; American Brachytherapy Society. The American Brachytherapy Society recommendations for brachytherapy of soft tissue sarcomas. Int $\mathbf{J}$ Radiat Oncol Biol Phys. 2001;49(4):1033-43.

111. Flamant F, Gerbaulet A, Nihoul-Fekete C, et al. Long-term sequelae of conservative treatment by surgery brachytherapy and chemotherapy for vulval and vaginal rhabdomyosarcoma in children. J Clin Oncol 1990; 8:1847-1853.

112. Nag S, Olson T, Ruymann F, et al. High dose rate brachytherapy in childhood sarcomas: a local control strategy presserving bone growth and function. Med Ped Oncol 1995;25:463-469.

113. Nag S, Tippin D, Ruymann FB. Long term morbidity in children treated with fractionated high dose rate brachytherapy for soft tissue sarcomas. J Pediatr Hematol Oncol 2003;25:448-452.

114. Nag S, Martínez-Monge R, Ruymann FB, Bauer C. Feasibility of intraoperative highdose rate brachytherapy to boost low dose external beam radiation therapy to treat pediatric soft tissue sarcomas. Med Ped Oncol 1998;31:79-85.

115. Nag S, Tippin D, Ruymann FB. Intraoperative highdose-rate brachytherapy for the treatment of pediatric soft tissue sarcomas. Int J Radiat Oncol Biol Phys 2001d;51:729-735.

116. Nag S, Kuske RR, Vicini F, Arthur DW, Zwicker RD. The American Brachytherapy Society recommendations for brachytherapy for carcinoma of the breast. Oncology 2001b; 15:195-207.

117. National Cancer Institute. Brachytherapy to Treat Cancer. Updated January 29, 2019.

118. Smith GL, Jiang J, Buchholz TA, et al. Benefit of adjuvant brachytherapy versus external beam radiation for early breast cancer: impact of patient stratification on breast preservation. Int J Radiat Oncol Biol Phys 2014;88(2):274-284.

119. Skowronek J. Current status of brachytherapy in cancer treatment - short overview. J Contemp Brachytherapy 2017;9(6):581589.

120. Skowronek J, Chicheł A. Brachytherapy in breast cancer: an effective alternative. Prz Menopauzalny 2014;13(1):48-55.

121. Svoboda VH, Kovarik J, Morris F. High dose-rate microselectron molds in the treatment of skin tumors. Int J Radiat Oncol Biol Phys 1995; 31:967-972.

122. Guix B, Finestres F, Tello J, Palma C. Treatment of skin carcinomas of the face by high-dose-rate brachytherapy and custommade surface molds. Int J Radiat Oncol Biol Phys 2000;47:95-102.

123. Parkin DM, Bray F, Ferlay J, Pisani P. Global cancer statistics, 2002. CA Cancer J Clin 2005;55(2):74-108.

124. Sirzén F, Kjellén E, Sörenson S, CavallinStåhl E. A systematic overview of radiation therapy effects in non-small cell lung cancer. Acta Oncol 2003;42(5-6):493-515.

125. Dagnault A, Ebacher A, Vigneault E, Boucher S. Retrospective study of 81 patients treated with brachytherapy for endobronchial primary tumor or metastasis. Brachytherapy. 2010;9(3):243-7.

126. Lo TC, Girshovich L, Healey GA, Beamis JF Jr, Webb-Johnson DC, Villanueva AG, Gray AW Jr, Wu TR. Low dose rate versus high dose rate intraluminal brachytherapy for malignant endobronchial tumors. Radiother Oncol. 1995;35(3):193-7.

127. Stout R, Burt P, Barber P. "Intraluminal radiotherapy", Advances in Radiation Oncology in Lung Cancer (JEREMIĆ, B., Ed.), Medical Radiology Radiation Oncology, Springer, Berlin and Heidelberg 2005;269-274.

128. Carvalho Hde A, Gonçalves SL, Pedreira W Jr, Gregório MG, de Castro I, Aisen S. Irradiated volume and the risk of fatal hemoptysis in patients submitted to high dose-rate endobronchial brachytherapy. Lung Cancer 2007;55(3):319-27.

129. Mehta M, Petereit D, Chosy L, Harmon M, Fowler J, Shahabi S, Thomadsen B, Kinsella T. Sequential comparison of low dose rate and hyperfractionated high dose rate endobronchial radiation for malignant airway occlusion. Int J Radiat Oncol Biol Phys 1992;23(1):133-9.

130. Reveiz L, Rueda JR, Cardona AF. Palliative endobronchial brachytherapy for non-small cell lung cancer. Cochrane Database Syst Rev. 2012;12:CD004284. 
131. Nag S, Scruggs GR. Clinical Aspects of High Dose Rate Brachytherapy, Lippincott Williams \& Wilkins, Philadelphia, PA, 2008.

132. Strassmann G, Walter S, Kolotas C, Heyd $\mathrm{R}$, Baltas $\mathrm{D}$, Debertshäuser $\mathrm{D}$, Nier $\mathrm{H}$, Tonus C, Sakas G, Zamboglou N. Reconstruction and navigation system for intraoperative brachytherapy using the flab technique for colorectal tumor bed irradiation. Int J Radiat Oncol Biol Phys. 2000;47(5):1323-9.

133. Merchant TE, Zelefsky MJ, Sheldon JM, et al. High-dose rate intraoperative radiation therapy for pediatric solid tumors. Med Ped Oncol 1998; 30:34-39.

134. Nag S, Gunderson L, Harrison L. Techniques of intraoperative radiation therapy vs. intraoperative high dose rate brachytherapy. In: Intraoperative irradiation: techniques and results. Gunderson LL, Willet CG,
Harrison LV, Calvo FA (eds) Humana Press, Totowa, NJ, 1999; 111-130.

135. Nag S, Hu KS. Intraoperative high-doserate brachytherapy. Surg Oncol Clin N Am 2003;12:1079-1097.

136. Nag S, Dally M, De la Torre M, et al. Recommendations for implementation of high dose rate 192 Ir brachytherapy in developing countries by the advisory group of international atomic energy agency. Radiother Oncol 2002; 64:297-308.

137. Li S, Frassica D, DeWeese T. A real-time image-guided intraoperative high dose-rate brachytherapy system. Brachytherapy 2003; 2:5-16.

138. Nag S, Cardenes $H$, Chang $S$, et al. Proposed guidelines for image-based intracavitary brachytherapy for cervical carcinoma: a report from the image-guided brachytherapy working group. Int J Radiat Oncol Biol Phys 2004; 60:1160-1172. 